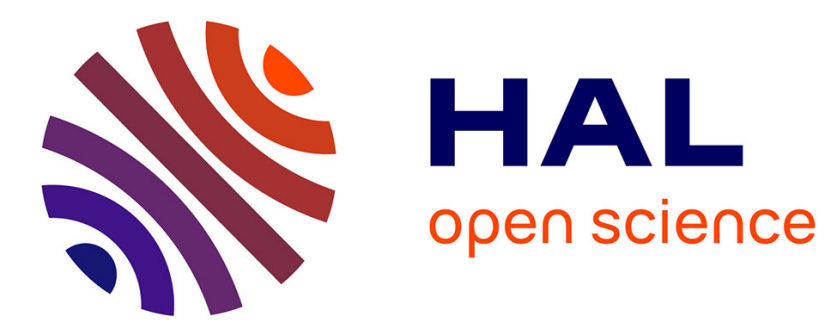

\title{
Reduced Dynamics of the Non-holonomic Whipple Bicycle
}

\author{
Frédéric Boyer, Mathieu Porez, Johan Mauny
}

\section{To cite this version:}

Frédéric Boyer, Mathieu Porez, Johan Mauny. Reduced Dynamics of the Non-holonomic Whipple Bicycle. Journal of Nonlinear Science, 2017, 10.1007/s00332-017-9434-x . hal-01717298

\section{HAL Id: hal-01717298 \\ https://hal.science/hal-01717298}

Submitted on 26 Feb 2018

HAL is a multi-disciplinary open access archive for the deposit and dissemination of scientific research documents, whether they are published or not. The documents may come from teaching and research institutions in France or abroad, or from public or private research centers.
L'archive ouverte pluridisciplinaire HAL, est destinée au dépôt et à la diffusion de documents scientifiques de niveau recherche, publiés ou non, émanant des établissements d'enseignement et de recherche français ou étrangers, des laboratoires publics ou privés. 


\title{
Reduced dynamics of the non-holonomic Whipple bicycle
}

\author{
Frédéric Boyer, Mathieu Porez and Johan Mauny ${ }^{1}$
}

\begin{abstract}
Though the bicycle is a familiar object of everyday life, modelling its full nonlinear three-dimensional dynamics in a closed symbolic form is a difficult issue for classical mechanics. In this article, we address this issue without resorting to the usual simplifications on the bicycle kinematics nor its dynamics. To derive this model, we use a general reduction based approach in the principal fiber bundle of configurations of the three-dimensional bicycle. This includes a geometrically exact model of the contacts between the wheels and the ground, the explicit calculation of the kernel of constraints, along with the dynamics of the system free of any external forces, and its projection onto the kernel of admissible velocities. The approach takes benefits of the intrinsic formulation of geometric mechanics. Along the path toward the final equations, we show that the exact model of the bicycle dynamics requires to cope with a set of non-symmetric constraints with respect to the structural group of its configuration fiber bundle. The final reduced dynamics are simulated on several examples representative of the bicycle. As expected the constraints imposed by the ground contacts, as well as the energy conservation are satisfied, while the dynamics can be numerically integrated in real time.
\end{abstract}

\section{Introduction}

Since its birth, the bicycle has drawn the attention of scientists from the pioneering treatises by $[37,7,8,39,14]$, to the most recent works of $[4,30,18]$, including the important steps by $[25,23,38,27,20]$ to name a few among the most significant ones. In particular, it is well known that the model of the rigid four-body bicycle with knife-edge wheels, introduced for the first time in the seminal works of Whipple [39], is a non-holonomic system, i.e. a system whose description of motions, requires more configuration coordinates than the number of its admissible velocities. Originally studied by Hertz [22], Appell [1] and Chaplygin [16], non-holonomic systems have recently aroused a new interest in the context of mobile robotics of planar wheeled vehicles such as the simple unicycle or the car-like platform [24, 13, 31]. Though sharing non-holonomy with these systems, the bicycle differs from them by the fact that contrarily to a simple unicycle, its shape-controlled locomotion dynamics cannot be fully described with a kinematic model but also require a further dynamic model. As such, the bicycle belongs to the less common class of dynamic non-holonomic systems which have been studied over the past years in the community of geometric mechanics [15], and geometric control [6], with applications to planar undulatory systems as the snake-board [34, 33, 32]. In this system, the locomotion is based on the transfer of kinetic momentums from its internal (shape) degrees of freedom to its external (net) ones, through non-sliding conditions imposed by the wheels [11]. In spite of being a dynamic nonholonomic locomotion system as the snake-board or the younger trikke [17], the bicycle differs from these undulatory systems by several characteristics which make it a system unique. Indeed, the wheels of the bicycle are not used for kinetic momentum transfers, but rather to ensure the self-stability, a property which is fundamental for control theory [28, 21, 2, 3], and which certainly explains the empirical success of the bicycle [26]. From the point of view of modeling, the bicycle being fundamentally three-dimensional, the derivation of its dynamics is much more difficult than usual non-holonomic planar systems. Beyond the geometric nonlinearities which unavoidably grow while progressing toward dynamics, the modelling difficulties arise from the early geometric and kinematic modelling stages. In particular, the position of the contact points

\footnotetext{
${ }^{1}$ F. Boyer, M. Porez and J. Mauny IMT Atlantique, LS2N, La Chantrerie 4, rue Alfred Kastler B.P. 20722 - 44307 Nantes Cedex 3 France. E-Mail: frederic.boyer@imt-atlantique.fr
} 
in a frame attached to the bicycle move with the bicycle configuration, so requiring a contact model which is almost systematically eluded in the literature on the topic. Another modelling difficulty is due to the unavoidable holonomic constraints modelling no-penetration nor lifting of the wheels along vertical. This introduces a kinematic loop coupling the attitude of the bicycle with its handlebar steering, and results in an implicit nonlinear algebraic system that cannot be solved explicitly for the purpose of coordinate reduction [36]. All these difficulties probably explain why most of the bicycle dynamic models proposed so far are based on simplified designs, inertial approximations and approximated kinematics. In particular, the complex kinematic coupling imposed by the above mentioned kinematic loop as well as the configurationdependency of the contact points, are mostly ignored and replaced by approximated decoupled kinematics where the position of contact points in the bicycle frame only depend on its design parameters $[38,21,9,10]$. Beyond these analytical approximated models, recent progresses in computational multibody system dynamics have provided a definitive solution to the fully nonlinear dynamics of the Whipple bicycle and proposed a benchmark [30], which has opened the way toward the explanation of the self-stability of the bicycle [26], an issue that has been highly debated throughout the history of bicycle dynamics.

In this article, we address the issue of modelling the exact nonlinear dynamics of the threedimensional Whipple bicycle. In contrast to previous works on the topic, this issue is addressed in the context of the geometric locomotion theory on principal fiber bundles as it has been developed over the past years in the field of geometric mechanics and robotics $[24,35,11]$. In this context, the dynamics of the bicycle are investigated on its configuration space $S E(3) \times S$, where $S E(3)$ stands for the special Euclidean Lie group of the bicycle net displacements in the three-dimensional ambient space, while $S$ is the shape space of its internal degrees of freedom $(\mathrm{DoF})$. Following a reduction approach presented in [9], the dynamics are first stated in $(s e(3) \times T S / S) \times(S E(3) \times S)$, and then projected onto $(\operatorname{ker}(A, B)) \times(S E(3) \times S)$, where $\operatorname{ker}(A, B)$ stands for the kernel of a set of constraints modelling the zero ground-wheel velocities conditions. These kinematic constraints include the no-penetrating nor lifting conditions, a choice which allows changing the analytically unfeasible coordinate reduction into the feasible velocity reduction. The position of the ground-wheel contact points are exact and obtained by the inversion of a set of algebraic conditions imposing the parallelism of the planes tangent to the wheels with that of the ground. The solutions of this contact problem being dependent on the position-orientation of the bicycle in $S E(3)$, the kinematic constraints are not symmetric (with respect to the structural group) along the fibers of $S E(3) \times S$. However, taking advantage of the intrinsic modelling in $S E(3)$ allows outsourcing all the artificial nonlinearities induced by the three-dimensional rigid body motions (as those introduced by the Euler angles usually used for modelling the 3D bicycle) to a set of reconstruction equations that can be numerically integrated. The approach gives at the end a closed symbolic form of the reduced dynamics of the bicycle whose solutions satisfy the constraints as soon as the dynamics are initialized in a configuration which is compatible with the two holonomic constraints of vertical no-penetrating nor lifting. The final equations are fully nonlinear and can be qualified of "geometrically exact" since they do not require any of the simplifications usually done to study this system. To validate the approach, these reduced dynamics are simulated on several examples with comparisons to a numerical benchmark recently proposed in [30]. As expected, the results obtained with our reduction-based approach match those of this numerical code and tend to show that a closed form of the fully nonlinear dynamics of the three-dimensional bicycle, at least for simple designs, could be in fact reachable. Finally, the aims of the article are two folds. Firstly, it provides a new (intrinsic) formulation of the bicycle dynamics. Secondly, it illustrates how some of the reduction techniques developed in recent years can be applied to the three-dimensional Whipple bicycle. 
The article is structured as follows. We start by giving the parametrization of a MMS and the notations we use in the article in section 2. The purpose of section 3 is to provide a general algorithm for deriving in a systematical manner, the reduced dynamics of a MMS with persistent point contacts. The next three sections consist of the application of this general algorithm to the case of a three-dimensional bicycle. The modelling of the bicycle starts with the geometric model of the contacts wheels-ground in section 4, continues with its kinematics (section 5), and ends with its dynamic model (section 6). These reduced dynamics are numerically tested in section 7 while the article ends by a conclusion (section 8 ).

\section{Parametrization of a MMS, application to the bicycle}

The bicycle is modelled as a rigid Mobile Multibody System, or MMS, interacting with a fixed rigid substrate through persistent ideal contacts. Such a system (see figure 1) is constituted of a sequence of $n+1$ rigid bodies $\mathcal{B}_{0}, \mathcal{B}_{1}, \ldots, \mathcal{B}_{n}$ interconnected through $n$ one DoF revolute joints parameterized by the vector $r=\left(r_{1}, \ldots, r_{n}\right)^{T}$ of relative angles around the joint axis between the bodies. The entire system moves in the ambient Euclidean space, or "physical space", endowed with a fixed orthonormed frame $\mathcal{F}_{e}=\left(O_{e}, s_{e}, n_{e}, a_{e}\right)$, named spatial frame. The body $\mathcal{B}_{0}$ stands for the reference body, i.e., for an arbitrarily distinguished body whose motions define the net motions of the MMS, and with respect to which, the motions of the other bodies define the shape time-variations of the MMS. Any MMS configuration can be defined by an instance of the vector $r=\left(r_{1}, \ldots, r_{n}\right)^{T}$, i.e., as a point on the manifold $S=\left(S^{1}\right)^{n}$, together with a position-orientation matrix $g \in S E(3)$ of $\mathcal{B}_{0}$ with respect to $\mathcal{F}_{e}$. The set $S E(3)$ is the Lie group of Euclidean displacements in $\mathbb{R}^{3}$, and the configuration space of the MMS is a trivial principal fiber bundle $\mathcal{C}=S E(3) \times S$, i.e., the product of a base manifold (here $S$ ), and of a structural group (here $S E(3))$, named the fiber. In $\mathcal{C}$, a configuration of the MMS is defined by $(g, r)$ and the map $(g, r) \mapsto(h g, r)$ for any $h \in S E(3)$, defines the (left) action of $S E(3)$ on $\mathcal{C}$ [5]. Time evolutions of $r \in \mathbb{R}^{n}$ define the motions of the MMS on the shape space $S$, while those of $g \in S E(3)$ define its rigid net motions. Any arbitrary motions of the MMS is a composition of these two types of motions. At any time $t$, the contacts are assumed to be exerted through a discrete set of geometric points defined as the intersection of the current configuration of the MMS with the substrate. In each of these points, the linear velocity with respect to the substrate is forced to be zero along a set of directions defined in $\mathcal{F}_{0}$.

In figure 2, the above general model is applied to the three-dimensional rigid bicycle of Whipple [39], i.e., a four-body MMS constituted of a frame $\mathcal{B}_{0}$, a fork $\mathcal{B}_{1}$ and two knife-edge wheels $\mathcal{B}_{2}$ and $\mathcal{B}_{3}$, all these bodies being connected together through $n=3$ revolute joints parameterized by $r=\left(r_{1}, r_{2}, r_{3}\right)^{T} \in \mathbb{R}^{3}, r_{j}$ measuring the time-dependent angle between $\mathcal{B}_{j-1}$ and $\mathcal{B}_{j}$. The system is in contact with a planar ground to which the spatial frame $\left(O_{e}, s_{e}, n_{e}, a_{e}\right)$ is fixed, with $s_{e}$ supporting the vertical axis. According to figure 2, $\mu$ denotes the (fixed) fork angle, and each body $\mathcal{B}_{j}$ is endowed with a mobile orthonormed frame $\mathcal{F}_{j}=\left(O_{j}, s_{j}, n_{j}, a_{j}\right)$ where the center $O_{j}$ coincides with the center of the joint $j$, and $a_{j}$ supports the joint axis. For any tensor $T_{j}$ related to a body $\mathcal{B}_{j},{ }^{i} T_{j}$ denotes the matrix of its components in the frame $\mathcal{F}_{i}$. To simplify the notations, the upper-left index is omitted when $\mathcal{F}_{j}=\mathcal{F}_{i}$, i.e., $T_{j}={ }^{j} T_{j}$. The position of a point $P$ in a frame $\mathcal{F}_{j}$ of origin $O_{j}$ will be denoted $p_{j}(P)$. Finally, time derivation is denoted by a 'dot'. 


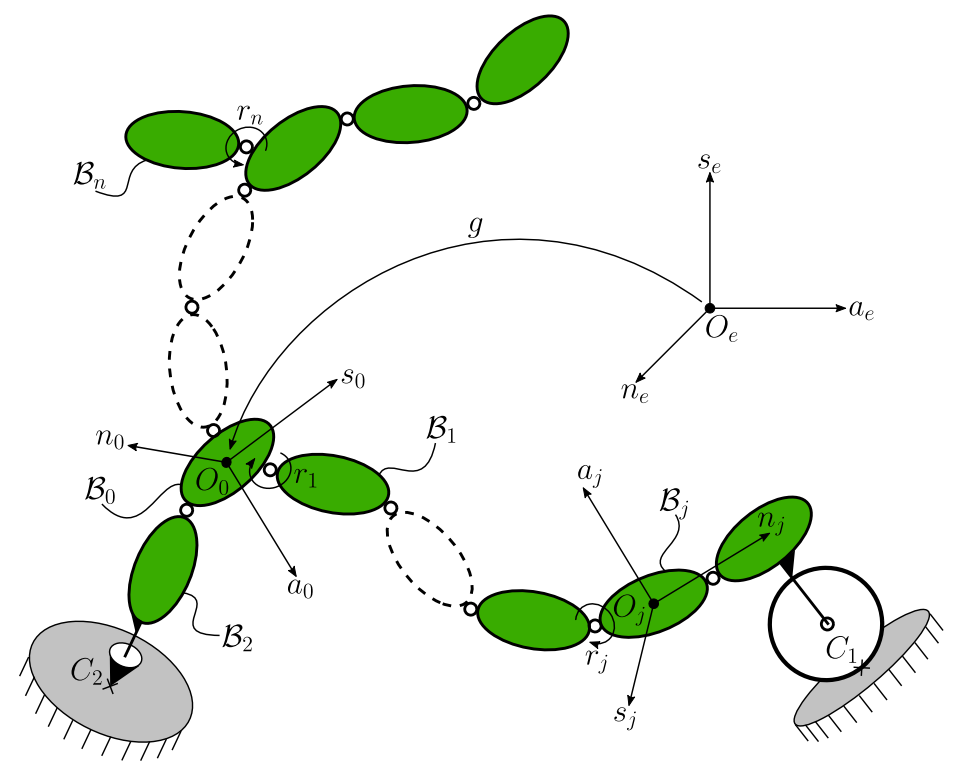

Figure 1: Mobile Multibody System subject to point contacts with a rigid substrate (the ground).

\section{Dynamics of a MMS subject to persistent contacts}

In this section, we present a reduction process of the dynamics of a MMS subject to constraints imposed by persistent point contacts with a rigid substrate. This process is introduced in [9] in the case when the system is possibly submitted to some imposed internal motions. Here, we will apply it to the case where only the internal forces can be imposed. This is the case of the bicycle when its handlebar and rear wheel are torque-controlled. Moreover, modelling the contacts exactly will need to extend the model of constraints introduced in [9] in a sense we will detail in the following.

\subsection{Dynamics of a constrained MMS in its configuration principal fiber bundle}

In all the subsequent developments a "hat" covering a vector $U$ will define a matrix $\hat{U}=U^{\wedge}$ whose definition depends on the dimension of $U$. Thus, if $U \in \mathbb{R}^{3}, \hat{U} \in \mathbb{R}^{3} \otimes \mathbb{R}^{3}$ denotes the skew-symmetric matrix such that $\hat{U} W=U \times W$ for $\forall W \in \mathbb{R}^{3}$. If $U=\left(V^{T}, W^{T}\right)^{T} \in \mathbb{R}^{6}$ with $V, W \in \mathbb{R}^{3}$, then:

$$
\hat{U}=\left(\begin{array}{cc}
\hat{W} & V \\
0 & 0
\end{array}\right) \in \mathbb{R}^{4} \otimes \mathbb{R}^{4}
$$

while if $U=\left(V^{T}, W^{T}\right)^{T} \in \mathbb{R}^{6+n}$, with $n$ the number of internal joints of the considered MMS, $V \in \mathbb{R}^{6}$, and $W \in \mathbb{R}^{n}$, then, the hat operator will be such that:

$$
\hat{U}=\left(\begin{array}{c}
\hat{V} \\
W
\end{array}\right) \in\left(\mathbb{R}^{4} \otimes \mathbb{R}^{4}\right) \times \mathbb{R}^{n}
$$

i.e., $\hat{U}$ represents the concatenation of a $4 \times 4$ matrix $(\hat{V})$ and a $n$-dimensional vector $(W)$.

According to our general model, the bicycle is considered as a constrained MMS subject to persistent point contacts which are assumed to be ideal. In its principal fiber bundle of configurations $\mathcal{C}=S E(3) \times S$, the paths $t \mapsto(g, r)(t)$ in $\mathcal{C}$ of any constrained MMS, are governed by 
dynamics of the general form:

$$
\left\{\begin{array}{l}
\left(\begin{array}{cc}
\mathcal{M} & M^{T} \\
M & m
\end{array}\right)\left(\begin{array}{l}
\dot{\eta} \\
\ddot{r}
\end{array}\right)=\left(\begin{array}{c}
f \\
Q
\end{array}\right)+\left(\begin{array}{l}
A^{T} \\
B^{T}
\end{array}\right) \lambda, \\
\dot{g}=g \hat{\eta} .
\end{array}\right.
$$

The bottom-equation of (3) stands for a kinematic model allowing to reconstruct the trajectory of the MMS in $\mathcal{C}$ from the time-evolution of its velocity $\left(\eta^{T}, \dot{r}^{T}\right)^{T} \in s e(3) \times T_{r} S$, itself governed by the top equation of (3). In (3), we find from left to right, the inertia matrix of the system in $\left(s e(3)^{*} \times \mathbb{R}^{n}\right) \otimes\left(s e(3) \times \mathbb{R}^{n}\right)$, the vector of accelerations in $s e(3) \times \mathbb{R}^{n}$, the vector of inertial, external and control forces which is detailed as:

$$
\left(\begin{array}{c}
f \\
Q
\end{array}\right)=\left(\begin{array}{c}
f_{\text {in }} \\
Q_{\text {in }}
\end{array}\right)+\left(\begin{array}{c}
f_{\text {ext }} \\
Q_{\text {ext }}
\end{array}\right)+\left(\begin{array}{c}
0_{6} \\
\tau
\end{array}\right),
$$

with $\left(f_{i n}^{T}, Q_{i n}^{T}\right)^{T}$ the vector of Coriolis and centrifugal forces, $\left(f_{\text {ext }}^{T}, Q_{\text {ext }}^{T}\right)^{T}$, that of external (except those exerted by the contact), and $\left(0_{6}^{T}, \tau_{1}, \tau_{2} \ldots \tau_{n}\right)^{T}$ the vector of control forces, all these forces being in $s e(3)^{*} \times \mathbb{R}^{n}$. Finally on the right hand side of (3), we find the vector of generalized forces (in $s e(3)^{*} \times \mathbb{R}^{n}$ ) imposed by the ideal contacts, where $\lambda \in \mathbb{R}^{m}$ is a vector of Lagrange multipliers modelling the reaction forces that are exerted in the physical space by the substrate onto the MMS through the contact points. These contacts are modelled by $m$ independent kinematic constraints of the general form:

$$
0_{m}=A(g, r) \eta+B(g, r) \dot{r},
$$

where $A$ et $B$ are some matrices (with $m=\operatorname{rank}(A, B)$ ), deduced from zero-velocity conditions between the substrate and the system in their contact points.

Remark 1: Practically, to derive the constraints (5), we first consider a each time, a set of $p$ contact points $C_{i}, i=1,2, \ldots, p$. These are geometric points defined as the intersection of the current configuration of the MMS with the substrate. These points are parameterized by their positions $p_{0}\left(C_{i}\right)$ in $\mathcal{F}_{0}$. In each of these points, the contact prevents the translations of the MMS with respect to the substrate, along $m_{i}$ directions noted $u_{l}\left(C_{i}\right), l=1,2, \ldots, m_{i} \leq 3$, where each $u_{l}$ is a unit vector of the three-dimensional physical space $\mathbb{R}^{3}$ expressed in $\mathcal{F}_{0}$. Thus, we start from the relations in the physical space:

$$
u_{l}^{T}\left(C_{i}\right) v\left(p_{0}\left(C_{i}\right)\right)=0, i=1,2, \ldots, p, l=1,2, \ldots, m_{i},
$$

in which, it suffices to introduce the position and velocity fields of the MMS in $\mathcal{F}_{0}$, noted $p_{0}$ and $v$, as functions of $(g, \eta, r, \dot{r})$, to obtain a set of $\sum_{i=1}^{p} m_{i}$ constraints on $S E(3) \times s e(3)^{*} \times T S^{*}$, from which we extract the expected $m$ independent constraints of (5).

Remark 2: The map pairing each system configuration with the $p$ couples $\left(p_{0}\left(C_{i}\right), u\left(C_{i}\right)\right)$ where $u\left(C_{i}\right)=\left(u_{1}\left(C_{i}\right), u_{2}\left(C_{i}\right), \ldots, u_{m_{i}}\left(C_{i}\right)\right)$, defines the geometric model of contacts of a MMS subject to persistent point contacts. It is formally defined as:

$$
\left(\begin{array}{c}
\left(p_{0}, u\right)\left(C_{1}\right) \\
\ldots \\
\left(p_{0}, u\right)\left(C_{p}\right)
\end{array}\right)=F_{c}(g, r) .
$$

Remark 3: In contrast to the context of [9], the $A$ and $B$ matrices of (5) possibly depend on $g$. When this is not the case, the constraints are said to be left-invariant. Physically, this means 
that they are not affected by any rigid transformation applied to the fixed frame of space (the ground frame in the case of the bicycle), or equivalently, they do not depend on the positionorientation $g$ of $\mathcal{B}_{0}$ in space, when they are expressed in terms of the $\mathcal{F}_{0}$-related net velocities here denoted $\eta=g^{-1} \dot{g}$. On the dual side, if $A$ and $B$ are $g$-dependent, the reaction forces of the right hand side (3), are no more left-invariant too. In the case of the bicycle, we will see that the constraints imposed by the wheel-ground contacts are not left-invariant in $S E(3)$ as soon as its frame is tilted and its handlebar is turned. Finally, let us note after remark 1, that this dependency of constraints with respect to $g$ can occur if and only if the directions $u\left(C_{i}\right)$ or/and the positions of the contact points in $\mathcal{F}_{0}$, i.e. $p_{0}\left(C_{i}\right)$, are themselves $g$-dependent. In the case of the bicycle, we will see that this is for the second of these reasons that the constraints (5) are not left-invariant. Finally, let us note that for the bicycle, the dependency on $g$ also occurs through the gravity external forces, i.e., in the $\left(f^{T}, Q^{T}\right)^{T}$ vector of (3).

\subsection{Reduced dynamics of a constrained MMS}

The dynamics of the mobile system can be reduced through the projection of (3) in the kernel of the constraints (5). Practically, this reduction consists firstly in calculating the kernel $\operatorname{ker}(A, B)$ of (5), which stands for the subspace of admissible velocities. Then, the velocities and accelerations are reexpressed using this subspace through the relations:

$$
\left(\begin{array}{l}
\eta \\
\dot{r}
\end{array}\right)=H(g, r)\left(\begin{array}{l}
\eta_{r} \\
\dot{r}_{r}
\end{array}\right),\left(\begin{array}{l}
\dot{\eta} \\
\ddot{r}
\end{array}\right)=H(g, r)\left(\begin{array}{l}
\dot{\eta}_{r} \\
\ddot{r}_{r}
\end{array}\right)+\dot{H}(g, r)\left(\begin{array}{l}
\eta_{r} \\
\dot{r}_{r}
\end{array}\right),
$$

with:

$$
\dot{H}(g, r)=\left(\frac{d}{d \epsilon}\right)_{\epsilon=0} H(g \exp (\epsilon \hat{\eta}), r+\epsilon \dot{r}) .
$$

Geometrically, the columns $H_{k}$ of $H(g, r)$ define a set of $n+6-m$ independent vector fields $H_{k}:(g, r) \in S E(3) \times S \mapsto H_{k}(g, r) \in \operatorname{se}(3) \times T_{r} S$ spanning $\operatorname{ker}(A, B)(g, r)$, and we write more concisely $H=\operatorname{ker}(A, B)$. The components of the MMS velocities in this set of vector fields, or distribution, are gathered in $\left(\eta_{r}^{T}, \dot{r}_{r}^{T}\right)^{T}$, which defines a new vector of $\mathbb{R}^{6+n-m}$ named the vector of reduced velocities. Secondly, we introduce (8) in (3) that we project onto the space of (virtual) velocities which, being compatible with the contacts, also satisfy (8-left). Then, since we have $H^{T}(A, B)^{T}=0$, we get the reduced dynamics in the form:

$$
\left(\begin{array}{cc}
\mathcal{M}_{r} & M_{r}^{T} \\
M_{r} & m_{r}
\end{array}\right)\left(\begin{array}{c}
\dot{\eta}_{r} \\
\ddot{r}_{r}
\end{array}\right)=\left(\begin{array}{c}
f_{r} \\
Q_{r}
\end{array}\right),
$$

Which have to be supplemented with the reduced reconstruction equation, obtained by using (2) and $g^{-1} \dot{g}=\eta$ in (8-left):

$$
\left(\begin{array}{c}
\dot{g} \\
\dot{r}
\end{array}\right)=\left(\begin{array}{c}
g \\
1
\end{array}\right)\left(H\left(\begin{array}{c}
\eta_{r} \\
\dot{r}_{r}
\end{array}\right)\right)^{\wedge}
$$

In (10), we have introduced the following reduced matrices:

$$
\begin{gathered}
\left(\begin{array}{cc}
\mathcal{M}_{r} & M_{r}^{T} \\
M_{r} & m_{r}
\end{array}\right)=H^{T}\left(\begin{array}{cc}
\mathcal{M} & M^{T} \\
M & m
\end{array}\right) H \\
\left(\begin{array}{c}
f_{r} \\
Q_{r}
\end{array}\right)=H^{T}\left(\left(\begin{array}{c}
f \\
Q
\end{array}\right)-\left(\begin{array}{cc}
\mathcal{M} & M^{T} \\
M & m
\end{array}\right) \dot{H}\left(\begin{array}{l}
\eta_{r} \\
\dot{r}_{r}
\end{array}\right)\right) .
\end{gathered}
$$

The above relations stand for the projection of the dynamics (3) into the kernel of the constraints $H$. Since $\lambda$ plays no role in these relations, they can be alternatively interpreted as the projection in $H$ of the dynamics of the system free of any contacts, or more concisely, of the free dynamics given by (3) in which $\lambda=0$. 
Remark 4: Equations (10) and (11) define a closed set of differential equations whose timeintegration from initial conditions gives the paths of a constrained MMS in its configuration space $\mathcal{C}=S E(3) \times S$. It is worth noting that they hold for both non holonomic and holonomic systems. However, while in the first case, the constraints (5) are non integrable, in the second, they derive from a set of $m$ geometric constraints $\Phi(g, r)=0_{m}$, according to:

$$
\dot{\Phi}=\left(\frac{d}{d \epsilon}\right)_{\epsilon=0} \Phi(g \exp (\epsilon \hat{\eta}), r+\epsilon \dot{r})=A(g, r) \eta+B(g, r) \dot{r}=0_{m} .
$$

As a result, it is worth noting here that replacing the geometric constraints by these kinematic ones is equivalent if and only if the dynamical system $(10,11)$ is integrated by starting in the constraint sub-manifold $\Phi(g, r)=0_{m}$ of $\mathcal{C}$, i.e., if $\Phi\left(g\left(t_{0}\right), r\left(t_{0}\right)\right)=0_{m}$ where $t_{0}$ is the initial instant of the motion. In this case, since the reconstruction equation (11) progresses along velocities lying in the tangent spaces to the constraint sub-manifold, the system will remain on it while more generally, it will evolve in another level set such that $\Phi(g, r)=\Phi\left(g\left(t_{0}\right), r\left(t_{0}\right)\right)$. This context will hold for the three-dimensional bicycle since the wheel-ground contacts impose both holonomic and non-holonomic constraints.

Remark 5: Although mathematically, the constraint sub-manifold defined by the $m$ holonomic constraints $\Phi(g, r)=0_{m}$ is preserved by $(10,11)$, numerically the approximative time integration of the reconstruction equation introduces a slight drift which makes progressively them violated. As this will be illustrated later on, in the case of the bicycle, this drift can be easily suppressed by adding a fast numerical component to the reconstruction equation (11). This corrective term will aim at forcing the two wheel-ground vertical (holonomic) constraints without perturbing the slow reduced bicycle dynamics.

Remark 6: The above reduction process can be generalized to several other contexts. For instance, when a part or all the internal shape variables, or the poses of a set of rigid obstacles, are prescribed through known time evolutions. In this case, the reduced kinematics (8-left) contain a supplementary part which is not governed by a dynamic model (as (10)) but by a kinematic one, accounting for the relations between the prescribed velocities and those of the MMS. Even more, for these systems, the number of prescribed shape variables may be such that $\operatorname{ker}(A, B)=0$. In this case, the MMS is entirely governed by a kinematic model under the restriction that the constraints fulfill some mobility conditions. This is for instance the case of nonholonomic platforms as the unicyle or the car-like platform [13].

Remark 7: The above reduction process stands for a modelling algorithm structured in three stages and several steps. The first stage, in one step, consists of deriving the geometric model of contacts (7). In the case of the bicycle, this corresponds to determine the points of the front and rear wheels in contact with the ground as a function of the bicycle configuration in $\mathcal{C}=S E(3) \times\left(S^{1}\right)^{3}$. The second stage starts with the derivation of the model of constraints (5), which stands for an implicit kinematic model. In a second step, an explicit model of the form (8left) is deduced through the calculation of the kernel of the constraints. The next stage deals with dynamics and start by the calculation of the model of the free dynamics, given by (3), in which the generalized reaction forces are removed (i.e., we force $\lambda=0$ ). Then, these free dynamics are reduced according to the projection formulae $(12,13)$. Finally, the reduced dynamic equations (10) governing the time evolution of the reduced velocities need to be supplemented with the reconstruction equations (11) where the explicit (reduced) kinematic model of the second stage appears. In the rest of the article, we apply this multistage approach to the bicycle of figure 2 . In this case, we shall see that the contacts introducing $m=6$ constraints of the form (5), the algorithm will automatically generate $6+n-m=3$ equations of the type (10), and $6+n=9$ reconstruction equations (11). 


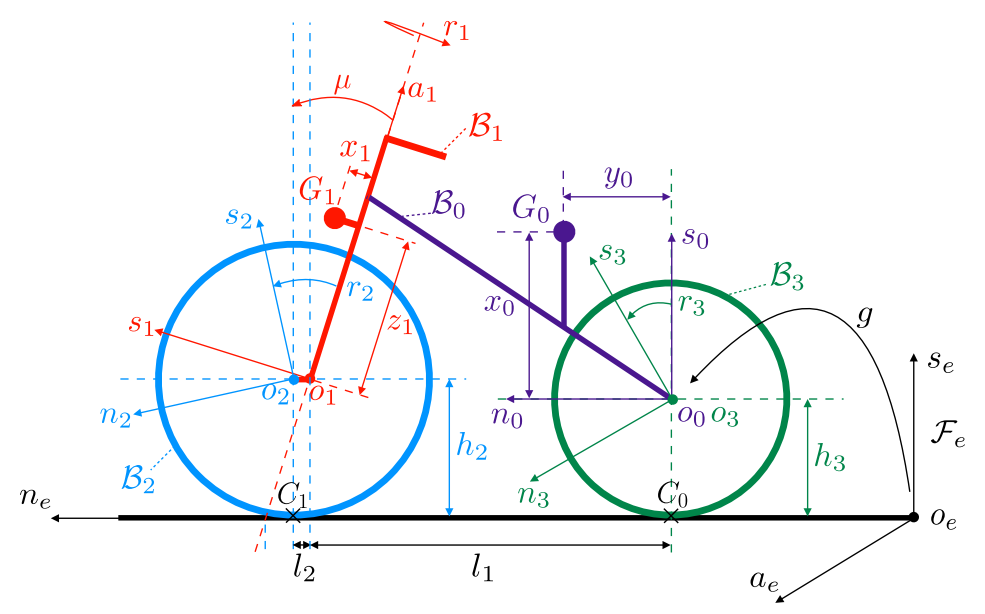

Figure 2: Frames and parameters of a three-dimensional bicycle. Throughout the article $\mathcal{B}_{0}, \mathcal{B}_{1}$, $\mathcal{B}_{2}, \mathcal{B}_{3}$ are named the "frame", the "fork", and the front and rear wheel respectively.

\section{Geometric model of contacts for a 3D bicycle}

In the wake of remark 2 , for the bicycle, in each of the two contact points, the wheel-ground velocities are prevented (i.e. forced to be zero) along any direction of $\mathcal{F}_{0}$. Thus, by "model of contacts" we mean a model allowing the calculation of the positions of contact points only, i.e., a function of the form:

$$
\left(\begin{array}{l}
p_{0}\left(C_{0}\right) \\
p_{0}\left(C_{1}\right)
\end{array}\right)=F_{c}(g, r),
$$

where $p_{0}\left(C_{0}\right)$ (respectively $p_{0}\left(C_{1}\right)$ ) represents the position in $\mathcal{F}_{0}$ of the contact point $C_{0}$ (respectively, of $C_{1}$ ) between the rear wheel (respectively, front wheel) with the planar ground. Finally, the purpose of what follows consists of deriving the expression of $F_{c}$.

\subsection{Preliminary calculation}

Due to the material symmetry of the two wheels, the model of contact (15) does not depend on $r_{2}$ and $r_{3}$. In other words, when deriving this model, one can consider an equivalent bicycle where the two material wheels are replaced by two geometric discs coinciding at each instant with the wheels. The rear and front wheels being attached to the frame $\mathcal{B}_{0}$ and the fork $\mathcal{B}_{1}$, these two discs will be named $\mathcal{D}_{0}$ and $\mathcal{D}_{1}$ respectively. They are related by a transformation noted $g^{\star}$, which referring to figure 2 , maps $\mathcal{F}_{0}=\left(O_{0}, s_{0}, n_{0}, a_{0}\right)$ onto $\left(O_{2}, s_{1}, n_{1}, a_{1}\right)$, i.e., onto a frame attached to the fork but centered on the hub of the front wheel. This transformation is detailed as:

$$
g^{\star}=\left(\begin{array}{cccc}
s \mu c r_{1} & -s \mu s r_{1} & c \mu & h_{2}-h_{3}+l_{2} s \mu c \mu\left(c r_{1}-1\right) \\
c \mu c r_{1} & -c \mu s r_{1} & -s \mu & l_{1}+l_{2}\left(c r_{1} c \mu^{2}+s \mu^{2}\right) \\
s r_{1} & c r_{1} & 0 & l_{2} c \mu s r_{1} \\
0 & 0 & 0 & 1
\end{array}\right) \text {, }
$$

which is configuration dependent through the shape variable $r_{1}$ only.

\subsection{Statement of the contact problem}

In this subsection, we address the following geometric problem. Let us consider the two geometric discs $\mathcal{D}_{0}$ and $\mathcal{D}_{1}$ previously defined. Knowing the transformation (16) that maps $\mathcal{D}_{0}$ onto $\mathcal{D}_{1}$, 
that we note more generically as:

$$
g^{\star}=\left(\begin{array}{cc}
R^{\star} & p^{\star} \\
0 & 1
\end{array}\right)=\left(\begin{array}{cccc}
R_{11}^{\star} & R_{12}^{\star} & R_{13}^{\star} & p_{1}^{\star} \\
R_{21}^{\star} & R_{22}^{\star} & R_{23}^{\star} & p_{2}^{\star} \\
R_{31}^{\star} & R_{32}^{\star} & R_{33}^{\star} & p_{3}^{\star} \\
0 & 0 & 0 & 1
\end{array}\right),
$$

as well as the position-orientation $g$ of $\mathcal{F}_{0}$ in space, that we detail as:

$$
g=\left(\begin{array}{cc}
R & p \\
0 & 1
\end{array}\right)=\left(\begin{array}{cccc}
R_{11} & R_{12} & R_{13} & p_{1} \\
R_{21} & R_{22} & R_{23} & p_{2} \\
R_{31} & R_{32} & R_{33} & p_{3} \\
0 & 0 & 0 & 1
\end{array}\right) .
$$

What are the positions in $\mathcal{F}_{0}$ of the points $C_{0} \in \partial \mathcal{D}_{0}$ and $C_{1} \in \partial \mathcal{D}_{1}$, through which passes a common plan $\mathcal{P}$, this plane being tangent to $\partial \mathcal{D}_{0}$ and $\partial \mathcal{D}_{1}$, and coincident with the ground plane?

\subsection{Calculation of the contact points}

We start by localizing $C_{0}$ and $C_{1}$ in the frame of the disc to which they belong, as follows:

$$
p_{0}\left(C_{0}\right)=\rho_{0}=\left(\begin{array}{c}
h_{3} c \alpha_{0} \\
h_{3} s \alpha_{0} \\
0
\end{array}\right),{ }^{1} p_{2}\left(C_{1}\right)=\rho_{1}=\left(\begin{array}{c}
h_{2} s \alpha_{1} \\
0 \\
h_{2} c \alpha_{1}
\end{array}\right),
$$

where, according to figure $2, h_{3}$ and $h_{2}$ denote the radius of the disc 0 and 1 whose center are $O_{0}$ and $O_{2}$ respectively, while their unit normal are $a_{0}$ and $n_{1}$ respectively. In (19), $\alpha_{0}$ (respectively, $\alpha_{1}$ ) denotes the angle between the vector $\overrightarrow{O_{0} C_{0}}$ (respectively, $\overrightarrow{O_{2} C_{1}}$ ), and $s_{0}$ (respectively, $a_{1}$ ), the two angles being oriented positively around $a_{0}$ and $n_{1}$ respectively. Once these vectors defined, we deduce from them, the tangent vectors to $\partial \mathcal{D}_{0}$ and $\partial \mathcal{D}_{1}$ in $C_{0}$ and $C_{1}$ respectively. These tangent vectors, noted $w_{0}$ and $w_{1}$, are given by:

$$
w_{0}=\frac{a_{0} \times \rho_{0}}{h_{3}}, w_{1}=\frac{n_{1} \times \rho_{1}}{h_{2}} .
$$

Now, we consider all the affine planes containing the affine line $\left(C_{0}, \vec{w}_{0}\right)$ and all the affine planes containing $\left(C_{1}, \vec{w}_{1}\right)$. Such planes are noted $\mathcal{P}_{0}$ and $\mathcal{P}_{1}$ respectively. All the planes $\mathcal{P}_{0}$ (respectively, all the planes $\mathcal{P}_{1}$ ), differ from each other by one rotation around $\left(C_{0}, \vec{w}_{0}\right)$ (around, $\left(C_{1}, \vec{w}_{1}\right)$ respectively). Each of these rotations is parameterized by one angle, named roll angle and noted $\phi_{0}$ for $\mathcal{D}_{0}$, and $\phi_{1}$ for $\mathcal{D}_{1}$. Each $\left.\phi_{i} \in\right]-\pi / 2, \pi / 2[$, is defined as the angle between the plane of the disc $\mathcal{D}_{i}$ with the normal $N_{i}$ to the contact plane $\mathcal{P}_{i}$ according to the context of figure 3 . In short, this is the angle of the rotation around $\left(C_{i}, \vec{w}_{i}\right)$ that must be applied to $\vec{N}_{i}$ to put it in the disc plane. With this choice, the roll angle is zero when the disc is orthogonal to its contact plane, and we have more generally:

$$
N_{0}=-s \phi_{0} a_{0}-c \phi_{0}\left(\rho_{0} / h_{3}\right), N_{1}=-s \phi_{1} n_{1}-c \phi_{1}\left(\rho_{1} / h_{2}\right)
$$

or again, in terms of components in the basis of the two discs:

$$
N_{0}=\left(\begin{array}{c}
-c \phi_{0} c \alpha_{0} \\
-c \phi_{0} s \alpha_{0} \\
-s \phi_{0}
\end{array}\right), N_{1}=\left(\begin{array}{c}
-c \phi_{1} s \alpha_{1} \\
-s \phi_{1} \\
-c \phi_{1} c \alpha_{1}
\end{array}\right)
$$



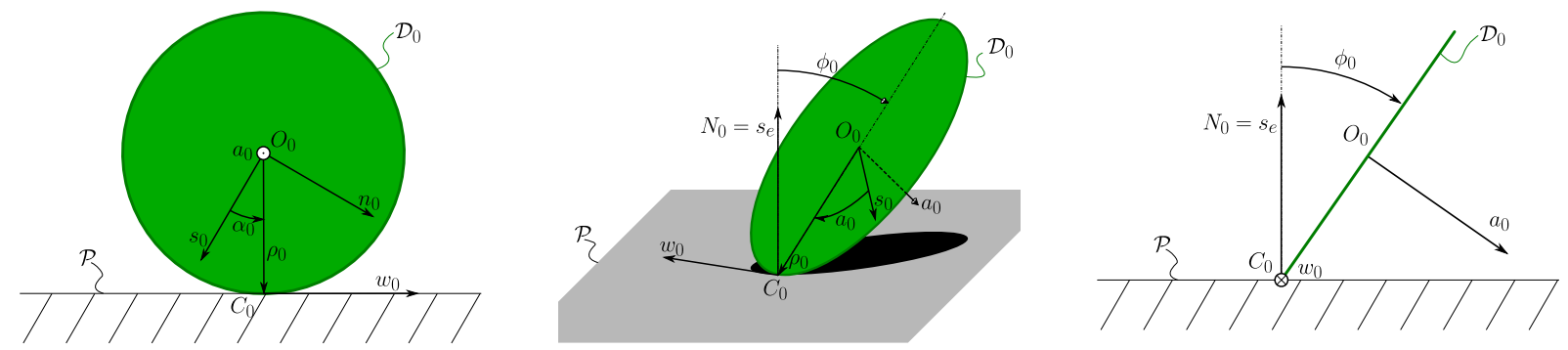

Figure 3: Parametrization of a disc (here the rear wheel) in contact with a plane (the ground).

Finally, we seek the conditions that $\left(\phi_{0}, \phi_{1}, \alpha_{0}, \alpha_{1}\right)$ have to satisfy in order to ensure $\mathcal{P}_{1}=$ $\mathcal{P}_{0}=\mathcal{P}$, where $\mathcal{P}$ is the ground plane to which the frame $\left(O_{e}, s_{e}, n_{e}, a_{e}\right)$ is attached, with $s_{e}$ its unit normal. To find out these conditions, it suffices to impose that the 3 planes are parallel with a zero altitude (along $s_{e}$ ) between them, i.e., we need to have $N_{0}={ }^{0} N_{1}={ }^{0} s_{e}$, and ${ }^{0} s_{e}^{T}\left(p_{0}\left(C_{0}\right)-p_{0}\left(O_{e}\right)\right)={ }^{0} s_{e}^{T}\left(p_{0}\left(C_{1}\right)-p_{0}\left(C_{0}\right)\right)=0$. These conditions can be detailed as the system of two orientation equations:

$$
-\left(\begin{array}{l}
R_{11} \\
R_{12} \\
R_{13}
\end{array}\right)=\left(\begin{array}{c}
c \phi_{0} c \alpha_{0} \\
c \phi_{0} s \alpha_{0} \\
s \phi_{0}
\end{array}\right),-\left(\begin{array}{l}
R_{11} \\
R_{12} \\
R_{13}
\end{array}\right)=\left(\begin{array}{lll}
R_{11}^{\star} & R_{12}^{\star} & R_{13}^{\star} \\
R_{21}^{\star} & R_{22}^{\star} & R_{23}^{\star} \\
R_{31}^{\star} & R_{32}^{\star} & R_{33}^{\star}
\end{array}\right)\left(\begin{array}{c}
c \phi_{1} s \alpha_{1} \\
s \phi_{1} \\
c \phi_{1} c \alpha_{1}
\end{array}\right),
$$

which ensures the 3 planes to be parallel, along with the two equations of position

$$
{ }^{0} s_{e}^{T}\left(\rho_{0}+R^{T} p\right)=0,{ }^{0} s_{e}^{T}\left(\rho_{0}-R^{\star} \rho_{1}-p^{\star}\right)=0,
$$

which ensure the altitudes between the 3 planes to be zero. Although (23) and (24) are both needed to ensure the three planes (ground and contact planes along the two wheels) to be the same, these two sets of equations (in position and orientation) have a different status in our modelling approach. The two scalar position equations (24) being of the general form:

$$
\Phi_{p}(g, r)=0_{2},
$$

they are two holonomic constraints that according to remark 4, are satisfied through the integration of the reduced dynamics as soon as it is started from initial conditions which satisfy them. On the other hand, the two equations of orientation (23) do not represent constraint equations, but stand for the expected model of contacts. In fact, they allow us to find the positions of $C_{0}$ and $C_{1}$ from the knowledge of $\left(g, r_{1}\right)$, what is always possible since (23) defines a set of 4 independent scalar conditions relating the 4 angles $\left(\phi_{0}, \phi_{1}, \alpha_{0}, \alpha_{1}\right)$. To solve (23), we start by considering the two first components of the first equation (from left to right) from which we extract:

$$
\alpha_{0}=\operatorname{atan} 2\left(-R_{12} ;-R_{11}\right),
$$

which needs $c \phi_{0}>0$, a condition systematically satisfied since $\left.\phi_{0} \in\right]-\pi / 2, \pi / 2[$. Moreover, (26) has no singularity since $R_{11}={ }^{e} s_{0}^{T} s_{e}$ is zero if and only if the rear wheel lies in the ground plane what is physically prevented. Applying the same computational process to the second equation of (23), one obtains the expression of the angle $\alpha_{1}$ :

$$
\alpha_{1}=\operatorname{atan} 2\left(-\left(R_{11}^{\star} R_{11}+R_{21}^{\star} R_{12}+R_{31}^{\star} R_{13}\right) ;-\left(R_{13}^{\star} R_{11}+R_{23}^{\star} R_{12}+R_{33}^{\star} R_{13}\right)\right),
$$

in which we introduce the expression (16) of $g^{\star}$, and obtain:

$$
\alpha_{1}=\operatorname{atan} 2\left(-c r_{1}\left(R_{11} s \mu+R_{12} c \mu\right)-s r_{1} R_{13} ; R_{12} s \mu-R_{11} c \mu\right) .
$$


Remark 8: In the context of the integration of the bicycle dynamics, knowing at each time the current value of $\left(g, r_{1}\right)$ which satisfies (24), the expressions (26) and (28) will be used at each instant of the simulation to calculate the two contact points $C_{0}$ and $C_{1}$. More formally, using (26) and (28) in (19), allows obtaining the expression of the model of contacts (15) where $F_{c}$ stands for a numerical or a symbolic function. Consequently, in all the following, these two points are assumed to be known and will be used in the next section to derive a kinematic model of the bicycle.

Remark 9: Though only $\alpha_{0}$ and $\alpha_{1}$ are required in the following dynamic formulation, let us note that solving the model of contacts (23) also gives the two roll angles $\phi_{0}$ and $\phi_{1}$. This is easily explainable by the fact that the information about the rear wheel roll is contained in $g$, while the geometric model between the two discs $g^{\star}$ suffices to deduce the roll angle of one disc from the knowledge of the other. To obtain the expression of $\phi_{0}$, it suffices to add the square of the two first components of (23-left). This provides $c \phi_{0}= \pm\left(\left(R_{12}\right)^{2}+\left(R_{11}\right)^{2}\right)^{1 / 2}$, that can be combined with the third component of the same equation, to obtain:

$$
\phi_{0}=\operatorname{atan} 2\left(-R_{13} ;\left(\left(R_{11}\right)^{2}+\left(R_{12}\right)^{2}\right)^{1 / 2}\right),
$$

which for the same reasons as above, has no singularity and $c \phi_{0}>0$. Finally, applying the same calculation to (23-right), we obtain:

$$
\begin{gathered}
\phi_{1}=\operatorname{atan} 2\left(R_{12}^{\star} R_{11}+R_{22}^{\star} R_{12}+R_{32}^{\star} R_{13} ;\right. \\
\left.\left(\left(R_{11}^{\star} R_{11}+R_{21}^{\star} R_{12}+R_{31}^{\star} R_{13}\right)^{2}+\left(R_{13}^{\star} R_{11}+R_{23}^{\star} R_{12}+R_{33}^{\star} R_{13}\right)^{2}\right)^{1 / 2}\right),
\end{gathered}
$$

that we can rewrite:

$$
\begin{gathered}
\phi_{1}=\operatorname{atan} 2\left(-s \mu s r_{1} R_{11}-c \mu s r_{1} R_{12}+c r_{1} R_{13} ;\right. \\
\left.\left[\left(c r_{1}\left(s \mu R_{11}+c \mu R_{12}\right)+s r_{1} R_{13}\right)^{2}+\left(c \mu R_{11}-s \mu R_{12}\right)^{2}\right]^{1 / 2}\right),
\end{gathered}
$$

where we accounted for (16).

Remark 10: Following remark 3 , the model of contacts $F_{c}$ being $g$-dependent, we will see that the kinematic constraints (5) of the bicycle are not left-invariant in $S E(3)$. Moreover, looking at the expressions (26) and (27) shows that this dependency is due to $\left(R_{11}, R_{12}, R_{13}\right)^{T}={ }^{0} s_{e}$, i.e. it naturally occurs through the ground vertical direction in the bicycle frame, which breaks the spatial symmetry. Thus, this loss of symmetry is related to the three dimensional character of the bicycle, and should not contradict the symmetry in the ground plane. This weaker symmetry can be exhibited by replacing $S E(3)$ by $S E(2)$ in the definition of our configuration space. This may be accomplished by reconsidering the parametrization of a disc in contact with the ground introduced in section 4.2, and to remark that the angles $\phi_{0}$ and $\alpha_{0}$ whose expressions as functions of $R$ are given by (29) and (26), are in fact the two last Euler-angles of the following sequence:

$$
R=\exp \left(\psi \hat{s_{e}}\right) \exp \left(\phi_{0} \hat{w}_{0}\right) \exp \left(\left(\pi-\alpha_{0}\right) \hat{a_{0}}\right),
$$

where $\psi \in\left[0,2 \pi\left[\right.\right.$ represents a yaw angle between $n_{e}$ and $w_{0}$ which is defined as:

$$
\psi=\operatorname{atan} 2\left(-R_{23} ; R_{32}\right),
$$

and $\left(\pi-\alpha_{0}\right) \in\left[0,2 \pi\left[\right.\right.$ stands for the pitch angle between the bicycle frame axis $n_{0}$ and the ground reference $w_{0}$. This remark allows introducing an alternative natural parametrization of the bicycle kinematics where the roll and pitch rotations of the above sequence are shifted 

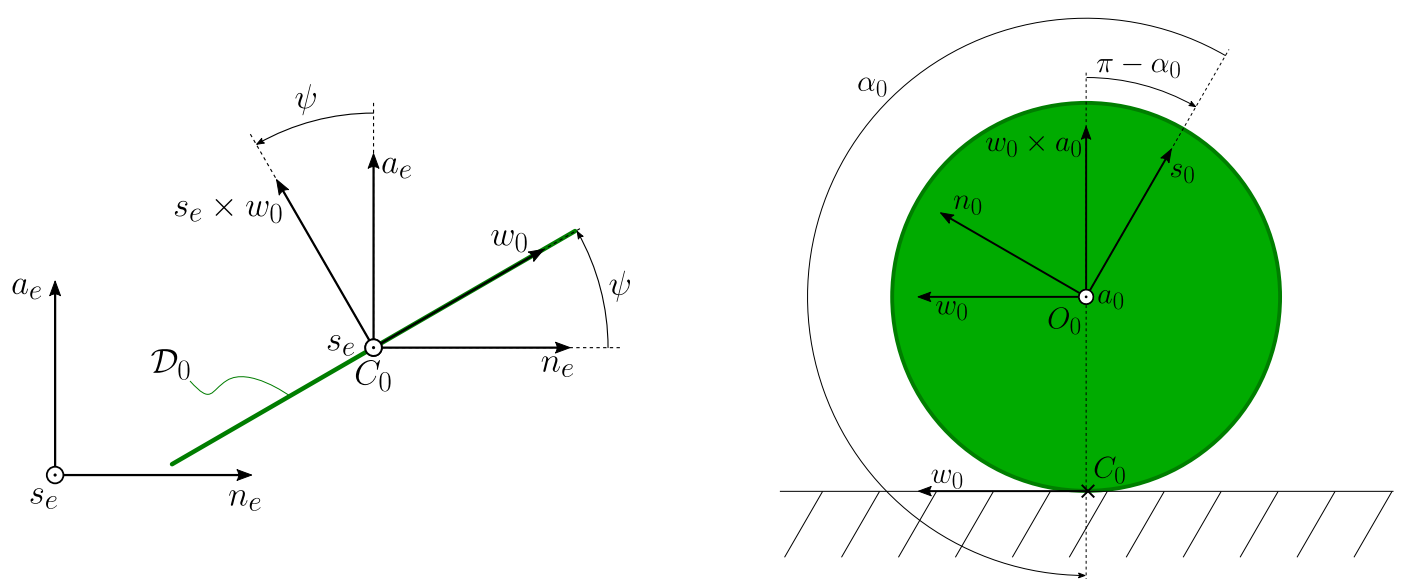

Figure 4: Top (left) and side view (right) of the rear wheel showing its yaw $(\psi)$ and pitch angles $\left(\pi-\alpha_{0}\right)$ in the contact frame.

from the fiber of $S E(3) \times S$ to the shape space of $S E(2) \times S$ where the points of $S$ are now parameterized by $\left(\phi_{0}, \pi-\alpha_{0}, r_{1}, r_{2}, r_{3}\right)$ and where the transformations of $S E(2)$ are simply:

$$
g=\left(\begin{array}{cc}
\exp \left(\psi \hat{s_{e}}\right) & p_{e}\left(C_{0}\right) \\
0 & 1
\end{array}\right)
$$

Thus, in this other parametrization, which is allowed by the fact that the two unilateral constraints of non penetration have been replaced by bilateral iso-altitude constraints, the reference frame is no more attached to the bicycle frame but is defined as the contact frame $\mathcal{F}_{0}=\left(C_{0}, s_{e}, w_{0}, s_{e} \times w_{0}\right)$ of figure 4 . It is remarkable that while in the three-dimensional parametrization, $\alpha_{0}$ stands for a kinematic observable of the model of contacts, in the planar parametrization, $\alpha_{0}$ stands for a generalized shape coordinate. However, this is just a question of interpretation as this is illustrated in figure 4 where depending upon whether the observer is attached to the rear wheel contact frame or to the bicycle frame, $\alpha_{0}$ measures the bicycle frame pitch with respect to the ground, or the rear wheel contact angle with respect to the bicycle frame, respectively.

Remark 11: Note that all our reduction approach can be applied to this other parametrization $(g, r)$ with $g \in S E(2)$ and $r=\left(\phi_{0}, \alpha_{0}, r_{1}, r_{2}, r_{3}\right)^{T}$. In particular, the bicycle has then $\operatorname{dim}(\mathcal{C})=$ $\operatorname{dim}(G)+\operatorname{dim}(S)=3+5=8$ DoFs, which are locally constrained by one rolling without slipping and one no-sliding constraint in $C_{0}$, along with one rolling without slipping, one no-sliding and one no-penetrating nor lifting, i.e., 3 constraints in $C_{1}$. Thus, one recovers $\operatorname{rank}(H)=8-5=3$ independent velocities. All these constraints are non-holonomic except the no-penetrating norlifting constraint in $C_{1}$ which is still given by (24-right) and can be detailed now, as:

$$
\left(\begin{array}{c}
-c \phi_{0} c \alpha_{0} \\
-c \phi_{0} s \alpha_{0} \\
s \phi_{0}
\end{array}\right)^{T}\left(\begin{array}{c}
s \mu c r_{1} h_{2} s \alpha_{1}+c \mu h_{2} c \alpha_{1}+h_{2} c \alpha_{0}+h_{2}-h_{3}+l_{2} s \mu c \mu\left(c r_{1}-1\right) \\
c \mu c r_{1} h_{2} s \alpha_{1}-s \mu h_{2} c \alpha_{1}+h_{2} s \alpha_{0}+l_{1}+l_{2}\left(c r_{1} c \mu^{2}+s \mu^{2}\right) \\
s r_{1} h_{2} s \alpha_{1}+l_{2} c \mu s r_{1}
\end{array}\right)=0,
$$

which is of the form $\Phi_{p}(g, r)=0_{1}$, and replaces (25) in our primary parametrisation. As regards the model of contacts (15), in this parametrisation, it takes the form $p_{0}\left(C_{1}\right)=F_{C}(g, r)$ where $p_{0}\left(C_{1}\right)$ now denotes the position of $C_{1}$ in the contact frame $\left(C_{0}, s_{e}, w_{0}, w_{0} \times s_{e}\right)$. Introducing 
(32) into (28), allows detailing this model as:

$$
\alpha_{1}=\operatorname{atan} 2\left(c r_{1} s\left(\alpha_{0}+\mu\right)-s r_{1} \tan \left(\phi_{0}\right) ; c\left(\alpha_{0}+\mu\right)\right) .
$$

Finally, integrating the reduced dynamics $(10,11)$ obtained with this parametrisation, allows reconstructing the full configuration $\left(g, \phi_{0}, \alpha_{0}, r_{1}, r_{2}, r_{3}\right)$, with $g$ given by (34). This configuration feeds the above model of contacts (36), which in turn feeds back the angle $\alpha_{1}$, so closing the formulation of the bicycle dynamics in $S E(2) \times S$.

Remark 12: In the literature on the bicycle, though the contact frame of the rear wheel is almost systematically uses as reference frame $\mathcal{F}_{0}$, the pitch angle $\alpha_{0}$, and the contact angle of the front wheel $\alpha_{1}$, which is an output of the model of contacts in the two parameterizations, are mostly ignored. Going further, using (36) in (35), or equivalently reconsidering the inversion of (23) to obtain $c \alpha_{1}$ and $s \alpha_{1}$ in terms of the configuration coordinates only, allows rewriting (35) as a complex nonlinear algebraic equation which cannot be solved explicitly for the purpose of removing $\alpha_{0}$ of the formulation. Such a nonlinear equation is derived in [36] and mentioned in [30] as a major obstacle to the explicit derivation of a closed form of bicycle dynamics. In the following, though this alternative parametrisation will be occasionally used, we will preferentially use our initial definition of $\mathcal{C}$ as $S E(3) \times S$. This choice has the advantage of removing the geometric nonlinearities that would be introduced by shifting the roll and pitch to the shape space, and to outsource them to the numerical integration of the reconstruction equation (11). Finally, it provides a more general formulation which opens the way for modelling the bicycle with unilateral contacts.

\section{$5 \quad$ Bicycle kinematics}

In this section, we derive the kinematic model of the bicycle on its configuration principal fiber bundle $\mathcal{C}=S E(3) \times S$. We start by deriving the model in the implicit form (5), from which an explicit kinematic model of the form (8) is deduced.

\subsection{Implicit bicycle kinematics}

In the following, we assume that the two angles $\alpha_{0}$ and $\alpha_{1}$ are known thanks to the model of contacts of the previous section (cf. remark 10). Then, considering the bicycle of figure 2, the kinematic constraints are derived by forcing the above vector relations:

$$
\begin{aligned}
& v\left(p_{0}\left(C_{1}\right)\right)=V+\Omega \times p_{0}\left(C_{1}\right)+\dot{r}_{1}^{0} a_{1} \times{ }^{0} p_{1}\left(C_{1}\right)+\dot{r}_{2}{ }^{0} a_{2} \times{ }^{0} p_{2}\left(C_{1}\right)=0, \\
& v\left(p_{0}\left(C_{0}\right)\right)=V+\Omega \times p_{0}\left(C_{0}\right)+\dot{r}_{3}{ }^{0} a_{3} \times{ }^{0} p_{3}\left(C_{0}\right)=0,
\end{aligned}
$$

where $\left(V^{T}, \Omega^{T}\right)^{T}=\eta$, and $v\left(p_{0}\left(C_{0}\right)\right)=0$ (respectively $v\left(p_{0}\left(C_{1}\right)\right)=0$ ) stands for the zero ground-wheel velocity at $C_{0}$ (respectively at $C_{1}$ ), both being expressed in the reference frame $\mathcal{F}_{0}$. These two vector equations provide $m=6$ scalar constraints that model the non-sliding, nonpenetrating nor lifting, and rolling without slipping conditions of the two wheels with respect to the ground. Simple algebra based on rigid body kinematics show that these constraints can be set in the general implicit form (5), which here takes the particular expression with 


$$
\begin{aligned}
V=\left(V_{1}, V_{2}, V_{3}\right)^{T}, \text { and } \Omega= & \left(\Omega_{1}, \Omega_{2}, \Omega_{3}\right)^{T}: \\
& V_{1}+A_{15} \Omega_{2}+A_{16} \Omega_{3}+B_{11} \dot{r}_{1}+B_{12} \dot{r}_{2}=0, \\
& V_{2}+A_{24} \Omega_{1}+A_{26} \Omega_{3}+B_{21} \dot{r}_{1}+B_{22} \dot{r}_{2}=0, \\
& V_{3}+A_{34} \Omega_{1}+A_{35} \Omega_{2}+B_{31} \dot{r}_{1}+B_{32} \dot{r}_{2}=0, \\
& V_{1}+A_{46} \Omega_{3}+B_{43} \dot{r}_{3}=0, \\
& V_{2}+A_{56} \Omega_{3}+B_{53} \dot{r}_{3}=0, \\
& V_{3}+A_{64} \Omega_{1}+A_{65} \Omega_{2}=0,
\end{aligned}
$$

where the components of $A$ and $B$ are defined as

$$
\begin{aligned}
& A_{26}=-A_{35}=\left(s \alpha_{1} c r_{1} h_{2}+\left(l_{2} c r_{1}-l_{2}\right) c \mu\right) s \mu+c \alpha_{1} h_{2} c \mu-h_{3}+h_{2}, \\
& A_{34}=-A_{16}=\left(s \alpha_{1} c r_{1} h_{2}+\left(l_{2} c r_{1}-l_{2}\right) c \mu\right) c \mu-c \alpha_{1} h_{2} s \mu+l_{2}+l_{1}, \\
& A_{15}=-A_{24}=l_{2} s r_{1} c \mu+s \alpha_{1} s r_{1} h_{2}, \\
& A_{56}=-A_{65}=h_{3} c \alpha_{0}, \\
& A_{64}=-A_{46}=h_{3} s \alpha_{0}, \\
& B_{11}=-s \mu s r_{1}\left(l_{2} c \mu+h_{2} s \alpha_{1}\right), \\
& B_{21}=-c \mu s r_{1}\left(l_{2} c \mu+h_{2} s \alpha_{1}\right), \\
& B_{31}=c r_{1}\left(l_{2} c \mu+h_{2} s \alpha_{1}\right) \\
& B_{12}=h_{2}\left(c r_{1} c \alpha_{1} s \mu-s \alpha_{1} c \mu\right), \\
& B_{22}=h_{2}\left(c r_{1} c \alpha_{1} c \mu+s \alpha_{1} s \mu\right), \\
& B_{32}=h_{2} s r_{1} c \alpha_{1} \\
& B_{43}=-h_{3} s \alpha_{0} \\
& B_{53}=h_{3} c \alpha_{0} .
\end{aligned}
$$

Remark 13: As expected by remark 11, the components of $A$ and $B$ depend on both the shape and the fiber variables. Going further into details, the above expressions depend explicitly on the shape through $r_{1}$, and implicitly on $g$, through the expressions of $\alpha_{0}$ and $\alpha_{1}$ given by (26) and (28) respectively. As announced by the remark 9, this second dependency corresponds to a loss of symmetry introduced by the geometric model of contacts which feeds the kinematic conditions (37) through the position vectors of $C_{0}$ and $C_{1}$.

Remark 14: As pointed out by remark 4 , two of the above kinematic constraints can be deduced through the derivation of the two holonomic constraints (24) of the form $\Phi_{p}(g, r)=0_{2}$, which impose each of the wheels to intersect the ground in one point. For confirmation of this, let us first time-differentiate (26) and (28). We obtain for $i=0,1$ :

$$
\dot{\alpha}_{i}=\frac{\dot{a}_{i} b_{i}-\dot{b}_{i} a_{i}}{a_{i}^{2}+b_{i}^{2}}
$$

where we introduced the notations:

$$
a_{0}=-R_{12}, b_{0}=-R_{11}, a_{1}=-c r_{1}\left(R_{11} s \mu+R_{12} c \mu\right)-s r_{1} R_{13}, b_{1}=R_{12} s \mu-R_{11} c \mu,
$$

as well as their time-derivatives:

$$
\begin{gathered}
\dot{a}_{0}=R_{11} \Omega_{3}-R_{13} \Omega_{1}, \dot{b}_{0}=R_{13} \Omega_{2}-R_{12} \Omega_{3}, \\
\dot{a}_{1}=\dot{r}_{1} s r_{1}\left(R_{11} s \mu+R_{12} c \mu\right)-s r_{1}\left(R_{11} \Omega_{2}-R_{12} \Omega_{1}\right)-\dot{r}_{1} c r_{1} R_{13}+c r_{1} s \mu \dot{b}_{0}+c r_{1} c \mu \dot{a}_{0}, \\
\dot{b_{1}}=\left(R_{13} \Omega_{1}-R_{11} \Omega_{3}\right) s \mu-\left(R_{12} \Omega_{3}-R_{13} \Omega_{2}\right) c \mu,
\end{gathered}
$$


which are obtained with the relation $\left(\dot{R}_{11}, \dot{R}_{12}, \dot{R}_{13}\right)^{T}=\left(R_{11}, R_{12}, R_{13}\right)^{T} \times \Omega$. Then, using the above expressions of $\dot{\alpha}_{0}$ and $\dot{\alpha}_{1}$ in the time differential of (24) gives:

$$
{ }^{0} s_{e}^{T} v\left(p_{0}\left(C_{0}\right)\right)=0,{ }^{0} s_{e}^{T}\left(v\left(p_{0}\left(C_{1}\right)\right)-v\left(p_{0}\left(C_{0}\right)\right)\right)=0 .
$$

As expected, (60) which is a linear combinations of the rows of (38-43), define two kinematic constraints which force the wheel-ground velocities to be zero along the ground vertical.

\subsection{Explicit bicycle kinematics}

Following the general modelling algorithm summarized by remark 6 , in this section we calculate the kernel of the kinematic constraints (38), with (44-56). This calculus stands for a generalized inversion of the implicit kinematic model of constraints. As a result, it can introduce some singularities that need to be treated apart. In the case of the bicycle such singularities do not exist, and the inversion of (38) gives after simple algebra detailed in Appendix 1, the explicit kinematic model of the bicycle in the form:

$$
\left(\begin{array}{c}
\eta \\
\dot{r}
\end{array}\right)=H(g, r)\left(\begin{array}{c}
\Omega_{2} \\
\dot{r}_{1} \\
\dot{r}_{3}
\end{array}\right),
$$

which corresponds to the instantiation of the general form (8-a) for the bicycle. Referring to the calculations of Appendix 1, $H$ can be detailed as:

$$
\left(\begin{array}{c}
V_{1} \\
V_{2} \\
V_{3} \\
\Omega_{1} \\
\Omega_{2} \\
\Omega_{3} \\
\dot{r}_{1} \\
\dot{r}_{2} \\
\dot{r}_{3}
\end{array}\right)=\left(\begin{array}{c}
H_{15} \\
H_{25} \\
H_{35} \\
H_{45} \\
1 \\
H_{65} \\
0 \\
H_{85} \\
0
\end{array}\right) \Omega_{2}+\left(\begin{array}{c}
H_{17} \\
H_{27} \\
H_{37} \\
H_{47} \\
0 \\
H_{67} \\
1 \\
H_{87} \\
0
\end{array}\right) \dot{r_{1}}+\left(\begin{array}{c}
H_{19} \\
H_{29} \\
H_{39} \\
H_{49} \\
0 \\
H_{69} \\
0 \\
H_{89} \\
1
\end{array}\right) \dot{r_{3}}
$$

whose components depend on $g$ through the two angles $\alpha_{0}$ and $\alpha_{1}$ and on $r$ through $r_{1}$.

\section{Bicycle dynamics}

According to remark 7, our general algorithm continues with the calculation of the free dynamics, i.e., the dynamics (3) with $\lambda=0$ from which we finally deduce the reduced dynamics of the bicycle. These reduced dynamics will be numerically integrated through several illustrative simulations in section 7 .

\subsection{Calculation of the free bicycle dynamics}

Among the several ways leading to the free dynamics, one can use the Poincare equations on the Lie group $G \times \mathbb{R}^{n}$ with the internal composition law $(g, r) \circ\left(g^{\prime}, r^{\prime}\right)=\left(g g^{\prime}, r+r^{\prime}\right)$ as in [12]. Though being conceptually straightforward, we here prefer to use a more computationally efficient approach based on the Newton-Euler recursive formulation of multibody systems [19]. In this approach, assuming that the bodies are labeled in increasing order from $\mathcal{B}_{0}$ to the tip of the branches ${ }^{2}$, and denoting $j$ the current body index and $i=a(j)$, that of its antecedent, the

\footnotetext{
${ }^{2}$ Note that the parametrization of figure 2 obeys these conventions.
} 
dynamics of the MMS of figure 1 can be restated in the alternative definition of the configuration space $\mathcal{C}=(S E(3))^{n+1}$

$$
\left\{\begin{array}{l}
\text { For } j=n, n-1, \ldots, 0: f_{j}=\mathcal{M}_{j} \dot{\eta}_{j}-f_{i n, j}-f_{e x t, j}+\sum_{k \mid a(k)=j} \operatorname{Ad}_{k}^{T} f_{j}, \\
\text { For } j=1,2,3 \ldots, n: \quad{ }^{e} \dot{g}_{0}={ }^{e} g_{0} \hat{\eta}_{0},{ }^{e} g_{j}={ }^{e} g_{i}{ }_{i} g_{j}\left(r_{j}\right), \eta_{j}=\operatorname{Ad}_{j} g_{i} \eta_{i}+\dot{r}_{j} \mathrm{~A}_{j},
\end{array}\right.
$$

whose the top row represents the balance of linear and angular kinetic momentums of each of the bodies isolated in the structure, while in the bottom row, we find from left to right the reconstruction equation of the reference body, along with the geometric and kinematic constraints imposed by the holonomic joints that connect the bodies. Going further into details, $\eta_{j} \in \operatorname{se}(3)$ denotes the velocity of $\mathcal{B}_{j}$ in $\mathcal{F}_{j}, \mathrm{~A}_{j} \in \operatorname{se}(3)$ is the unit velocity supported by the axis of the $j^{\text {th }}$ joint. $\mathcal{M}_{j}$ is the inertia matrix of $\mathcal{B}_{j}$ in $s e(3) \otimes s e(3)^{*}$ while $f_{j}, f_{i n, j}, f_{\text {ext }, j} \in \operatorname{se}(3)^{*}$, denote the inter-body wrench exerted by $\mathcal{B}_{j-1}$ onto $\mathcal{B}_{j}$, that of inertial (Coriolis and centrifugal) forces, and the wrench of external (gravity) forces exerted on body $j \cdot \operatorname{Ad}_{g}$ is the adjoint map of any $g \in S E(3)$ on $s e(3)$, and in the above context $\operatorname{Ad}_{g_{i}}$ allows shifting a velocity in $s e(3)$ from $\mathcal{F}_{i}$ to $\mathcal{F}_{j}$. Time differentiating the kinematic constraints gives the further equations relating the accelerations of consecutive bodies in the chain:

$$
\text { For } j=1,2, \ldots, n: \dot{\eta}_{j}=\operatorname{Ad}_{g_{i}} \dot{\eta}_{i}+\zeta_{j}+\ddot{r}_{j} \mathrm{~A}_{j},
$$

where $\zeta_{j}=\dot{A} d_{j} \eta_{i} \eta_{i}$ is detailed in Appendix 2. Now we consider the top row of (63) for $j=0$, and its projection onto $\mathrm{A}_{j}$ for $j \neq 0$, where let us remark that $\tau_{j}=\mathrm{A}_{j}^{T} f_{j}$. Then, using recursively (64) and (63-top), one can remove all the $f_{j}$ and $\dot{\eta}_{j}$ for $j \neq 0$, from these equations according to an elimination process detailed in Appendix 2. At the end we obtain an inverse dynamic model in the form:

$$
\left(\begin{array}{c}
f_{0} \\
\tau
\end{array}\right)=\left(\begin{array}{cc}
\mathcal{M} & M^{T} \\
M & m
\end{array}\right)\left(\begin{array}{c}
\dot{\eta}_{0} \\
\ddot{r}
\end{array}\right)-\left(\begin{array}{c}
f_{\text {in }} \\
Q_{\text {in }}
\end{array}\right)-\left(\begin{array}{c}
f_{\text {ext }} \\
Q_{\text {ext }}
\end{array}\right),
$$

where $f_{0}$ and $\tau=\left(\tau_{1}, \tau_{2}, \ldots, \tau_{n}\right)^{T}=\left(\mathrm{A}_{1}^{T} f_{1}, \mathrm{~A}_{2}^{T} f_{2}, \ldots, \mathrm{A}_{n}^{T} f_{n}\right)^{T}$ represent a $\mathcal{B}_{0}$-related wrench, and a vector of joint torques that should be applied to the MMS in order to move it with $\left({ }^{e} g_{0}, \eta_{0}, \dot{\eta}_{0}, r, \dot{r}, \ddot{r}\right)$. Since $\left({ }^{e} g_{0}, \eta_{0}, \dot{\eta}_{0}, r, \dot{r}, \ddot{r}\right)=(g, \eta, \dot{\eta}, r, \dot{r}, \ddot{r})$, the above mentioned recursive elimination provides at the end, the expected expressions of all the matrices of the free dynamics (3) with $\lambda=0$. These expressions are detailed in Appendix 2 in the case of the bicycle.

\subsection{Reduced bicycle dynamics}

Applying the projection relations $(12,13)$ to the above free dynamics gives the expected reduced dynamics:

$$
\left(\begin{array}{c}
\dot{\eta}_{r} \\
\ddot{r}_{r} \\
\dot{g} \\
\dot{r}
\end{array}\right)=\left(\begin{array}{c}
\left(\begin{array}{cc}
\mathcal{M}_{r} & M_{r}^{T} \\
M_{r} & m_{r}
\end{array}\right)^{-1}\left(\begin{array}{c}
f_{r} \\
Q_{r}
\end{array}\right) \\
\left(\begin{array}{c}
g \\
1
\end{array}\right)\left(H\left(\begin{array}{c}
\eta_{r} \\
\dot{r}_{r}
\end{array}\right)\right)^{\wedge}
\end{array}\right) .
$$

Referring to remarks 4 and 7 , these dynamics have to be initialized with any reduced state $\left(g, r, \eta_{r}, \dot{r}_{r}\right)(t=0)$ whose initial configuration satisfies (25). Among these compatible configurations, the simplest one is given by that of figure 2, i.e., it is defined by a pair $(g, r)$ with $r=\left(0, r_{2}, r_{3}\right), r_{2}$ and $r_{3}$ being arbitrary, and $g=(R, p)$, with $R=1_{3}, p=\left(h_{3}, y, z\right)$ and $y$ and $z$ arbitrary. This simple configuration being also statically balanced, it will be systematically used to initialize the dynamic simulations of section 7 . 
Remark 15: To find other compatible configurations, one can start from the simple configuration of figure 2, and make the bicycle tilt and roll on the ground using the kinematic part of the model only:

$$
\left(\begin{array}{c}
\dot{g} \\
\dot{r}
\end{array}\right)=\left(\begin{array}{c}
g \\
1
\end{array}\right)\left(H(g, r)\left(\begin{array}{c}
\Omega_{2} \\
\dot{r}_{1} \\
\dot{r}_{3}
\end{array}\right)\right)^{\wedge} .
$$

In fact, this model can be interpreted as a kinematic controlled system where $\left(\Omega_{2}, \dot{r}_{1}, \dot{r}_{3}\right)$ stands for a set of control inputs. Then, it is straightforward to find out any other compatible configuration by integrating (67) from the initial configuration of figure 2 while $\dot{r}_{3}$ is fixed to zero. For instance, one can impose a sequence of two manoeuvres. The first manoeuver consists of turning the handlebar from $r_{1}=0$ to a desired angular position $r_{1 d}$ with $\Omega_{2}=0$. The second consists of maintaining $r_{1}=r_{1 d}$ while tilting the frame by applying the control $\Omega_{2}=K_{\phi}\left(\phi_{0}-\phi_{0 d}\right)$ in (67), with $\phi_{0 d}$ a desired value of the rear wheel roll angle. Alternatively, one can directly impose $\dot{r}_{3}=0, \dot{r}_{1}=K_{1}\left(r_{1}-r_{1 d}\right)$, and $\Omega_{2}=K_{\phi}\left(\phi_{0}-\phi_{0 d}\right)$ in (67). These two control (manoeuvres or continuous) both allow steering (67) from the compatible configuration of figure 2 to any other (satisfying $\left.\Phi_{p}(g, r)=0_{2}\right)$ such that $r_{1}=r_{1 d}$ and $\phi_{0}=\phi_{0 d}$. All along the time integration of (67) controlled by this feedback law, the two angles $\alpha_{0}$ (which parameterizes the frame pitch), and $\alpha_{1}$ (which parameterizes the contact point of the front wheel), change with the configuration $g$ up to reach their final value.

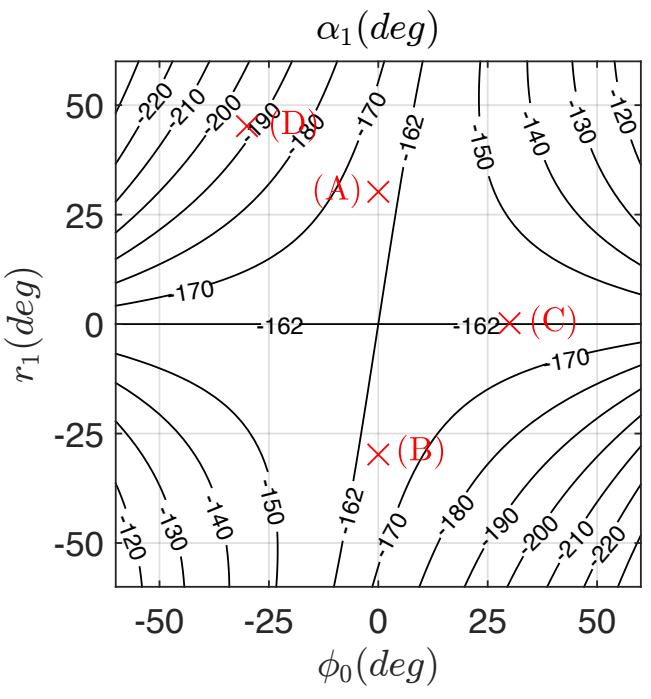

Figure 5: Portrait of iso-values of the front wheel contact angle $\alpha_{1}$ (in degrees) in the plane $\left(\phi_{0}, r_{1}\right)$.

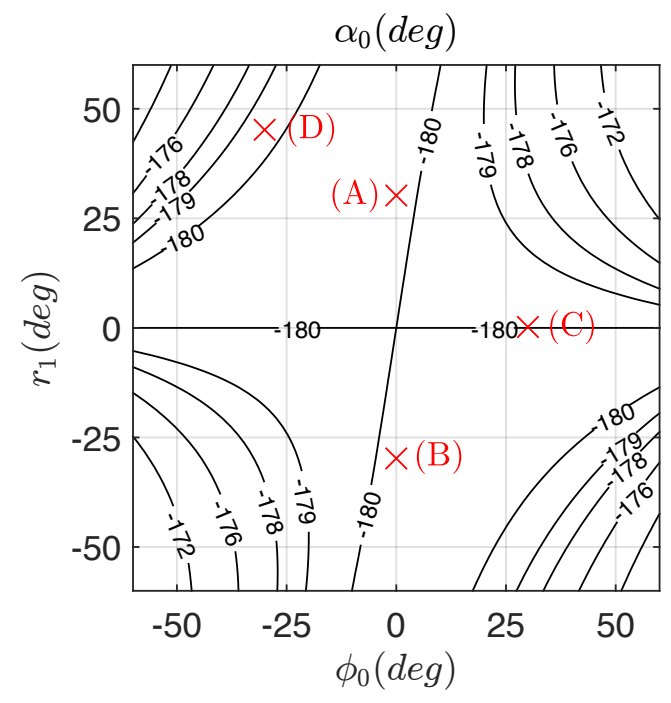

Figure 6: Portrait of iso-values of the rear wheel contact angle $\alpha_{0}$ (in degrees) in the plane $\left(\phi_{0}, r_{1}\right)$.

Remark 16: The portraits of figures 5 and 6 show the iso-values of $\alpha_{1}$ and $\alpha_{0}$ as functions of the roll angle $\phi_{0}$ and the steering handlebar $r_{1}$, both being numerically calculated after convergence of the above kinematic control law. For the purpose of illustration, the points A, B, C, D plotted in these portraits indicate four particular configurations drawn in figure 9 . Note that the middle point of portraits 5 and 6 corresponds to the case when the bicycle is flat and vertical, i.e., when it is in the configuration of figure 2 in which $\alpha_{1}=\pi+\mu$ (here the fork angle is set to $\mu=\pi / 10$ $\mathrm{rad}$ ). Then, shifting along the vertical $\phi_{0}=0$ of these portraits, by increasing $r_{1}$, corresponds to turn the handlebar on the left while maintaining the bicycle vertical. This rotation makes the contact points $C_{0}$ and $C_{1}$ move frontward along the rear and front wheel periphery, while 


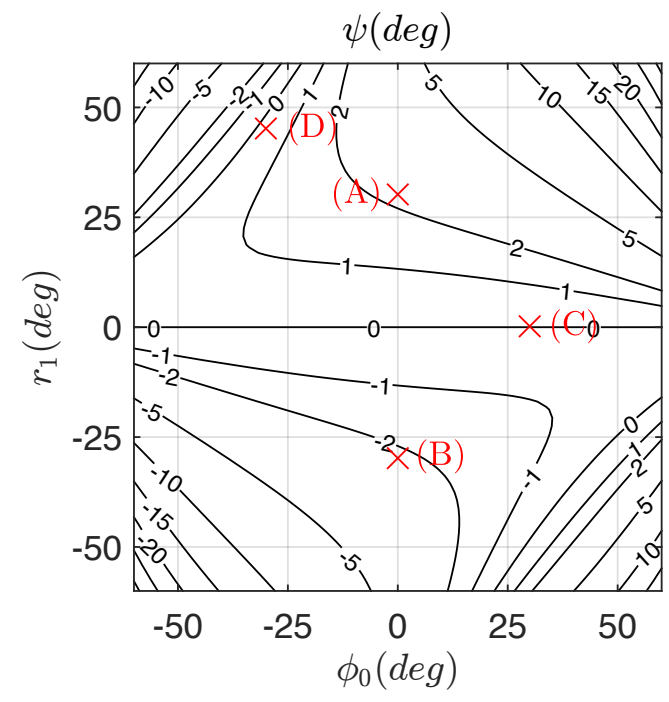

Figure 7: Portrait of iso-values of the yaw angle $\psi$ (in degrees) in the plane $\left(\phi_{0}, r_{1}\right)$.

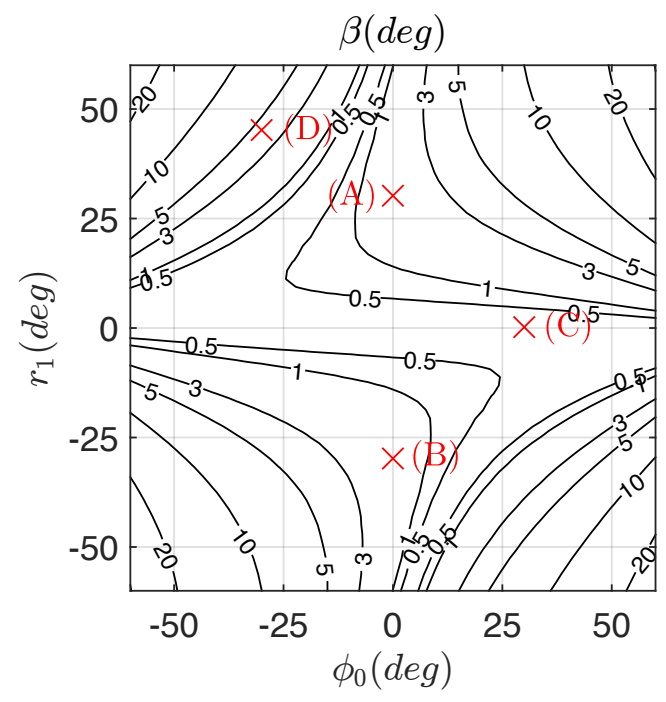

Figure 8: Portrait of iso-values of $\beta$ (in degrees) in the plane $\left(\phi_{0}, r_{1}\right)$.

an angle between the line of contact points $\overrightarrow{C_{0} C_{1}}$ and the rear wheel contact vector $\vec{w}_{0}$ appears and growths with $r_{1}$ as this is illustrated by the snapshots $\mathrm{A}$ and $\mathrm{B}$ of figure 9 which display the top views to the bicycle when $\phi_{0}=0, r_{1}=30^{\circ}(\mathrm{A})$, and $r_{1}=-30^{\circ}(\mathrm{B})$. Now, descending along $\phi_{0}=0$ in the portraits, i.e., rotating the handlebar on the right while maintaining the bicycle vertical, the contact points $C_{0}$ and $C_{1}$ move in the same way along the wheels periphery, while $\overrightarrow{C_{0} C_{1}}$ and $\overrightarrow{w_{0}}$ transform symmetrically with respect to the initial flat configuration. In particular, when the bicycle is vertical and such that $\mu=0$ (its fork angle is zero), then $C_{0}$ and $C_{1}$ become both independent of $r_{1}$, and $\alpha_{0}$ and $\alpha_{1}$ remain fixed to their initial value $\pi$ and $\pi+\mu=\pi$ along the vertical $\phi_{0}=0$. In this particular case, the slop of the straight iso-values of the portraits 5 and 6 with respect to the vertical axis $\phi_{0}=0$, are zero, and the two portraits are straightened. Now shifting along the line $r_{1}=0$ corresponds to configurations where the bicycle is flat but tilted with respect to the ground normal as this is illustrated on the snapshot $\mathrm{C}$ of figure 9 for which $\phi_{0}=30^{\circ}$ and $r_{1}=0$. In this case, the two contact points $C_{0}$ and $C_{1}$ no longer move along the wheels periphery nor on the ground. Along any oblique straight line drawn on the portraits, these two tendencies combine as this is illustrated on the snapshot D of figure 9 for which $\phi_{0}=-30^{\circ}$ and $r_{1}=45^{\circ}$. Finally, figures 7 and 8 display the portraits of the yaw angle $\psi$ and the angle $\beta$ between the line of contact points and that of the rear wheel in the $\left(\phi_{0}, r_{1}\right)$ plane. As expected, these plots show how the rear wheel rotates around $C_{0}$, and how $\overrightarrow{C_{0} C_{1}}$ deviates from $\vec{w}_{0}$ when the bicycle is tilted and its handlebar is turned. Finally, note also that since $\pi-\alpha_{0}$ measures the pitch of the bicycle frame, the portrait of figure 6 also shows how this angle slightly varies with the roll and handlebar angles.

Remark 17: The calculation of the reduced inertia forces (13) requires to derive $\dot{H}$, which involves the most complex expressions handled by the model. Since in our model of the contacts, the $g$-dependency appears through the two angles $\alpha_{0}$ and $\alpha_{1}$, the general formula (9) can be simply written as:

$$
\dot{H}=\left(\frac{\partial H}{\partial \alpha_{0}}\right) \dot{\alpha_{0}}+\left(\frac{\partial H}{\partial \alpha_{1}}\right) \dot{\alpha_{1}}+\left(\frac{\partial H}{\partial r_{1}}\right) \dot{r_{1}}
$$

where $\dot{\alpha}_{0}$ and $\dot{\alpha}_{1}$ are given by $(57,58,59)$. 

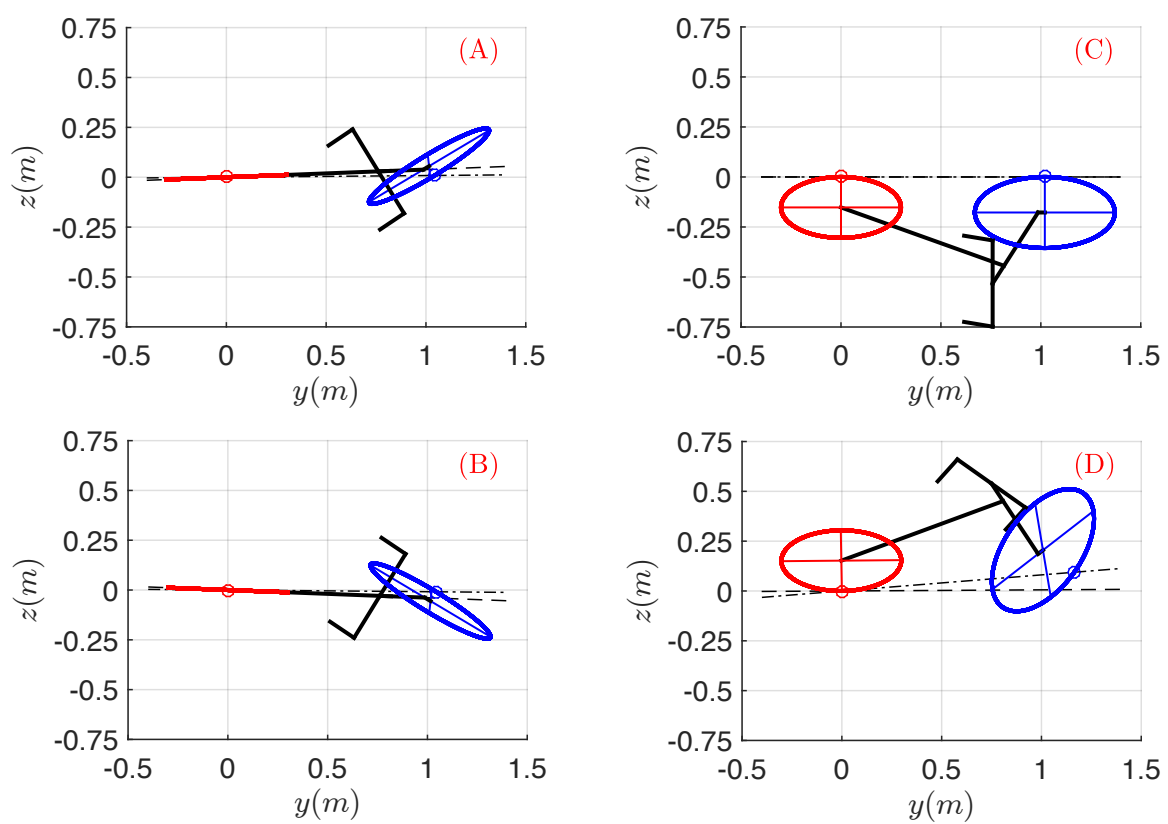

Figure 9: Top views to the bicycle when $\left(\phi_{0}, r_{1}\right)=(0,30)(\mathrm{A}),(0,-30)(\mathrm{B}),(30,0)(\mathrm{C})$ and $(-30,45)$, all angles being in degrees. (--) line of contact points on the ground. $(-\cdot-)$ projected line of the rear-wheel plane onto the ground.

\section{Simulations}

In this section, we apply our modelling approach to the bicycle of figure 2 , fed by the 26 parameters listed in table 1 which correspond to the benchmark bicycle proposed in [30], but translated in our modelling framework. Apart from the geometric parameters introduced before, in this table, $m_{0}$ (respectively, $\left.m_{1}\right)$ and $\left(x_{0}, y_{0}, 0\right)^{T}$ (respectively $\left.\left(x_{1}, 0, z_{1}\right)^{T}\right)$ denote the mass and the position of the mass center $G_{0}$ (respectively $G_{1}$ ) of the bicycle frame (respectively, of its fork) in $\mathcal{F}_{0}$ (in $\mathcal{F}_{1}$ ). The two matrices,

$$
I_{0}=\left(\begin{array}{ccc}
I_{0 x x} & I_{0 x y} & 0 \\
I_{0 x y} & I_{0 y y} & 0 \\
0 & 0 & I_{0 z z}
\end{array}\right), I_{1}=\left(\begin{array}{ccc}
I_{1 x x} & 0 & I_{1 z x} \\
0 & I_{1 y y} & 0 \\
I_{1 z x} & 0 & I_{1 z z}
\end{array}\right)
$$

denote the matrices of angular inertia of the frame and fork with respect to their mobile frame $\mathcal{F}_{0}$ and $\mathcal{F}_{1}$. The front and rear wheels are modelled as two homogeneous discs of mass $m_{2}$ and $m_{3}$. Their center of mass is located on the wheel hubs and their inertia matrix is diagonal with for the front wheel (for the rear wheel), an axial inertia moment noted $I_{2 z z}\left(I_{3 z z}\right)$ and radial inertia moments $I_{2 x x}=I_{2 y y}\left(I_{3 x x}=I_{3 y y}\right.$ respectively). Applying the above algorithm gives the reduced dymanic model in symbolic form. Using a quaternion parametrization for reconstruction, the model is time-integrated numerically in Matlab with a predictor-corrector method (a fourthorder explicit method for the prediction step and a fifth-order implicit method for the correction step). With no optimization, the symbolic model requires a few thousand of basic operations $(=,-,+, \times, /)$. At the end, the bicycle can be simulated in real time (with Matlab), on a intel i7 CPU @ $3 \mathrm{GHz}$ (as example $5 \mathrm{~s}$ of simulation takes less than $2.6 \mathrm{~s}$ of computation with a time step of $0.005 \mathrm{~s}$ ). Note that all subsequent simulations are illustrated by videos attached to this manuscript. 


\subsection{Kinematic reconstruction}

Following remark 4, the solutions of (66) should preserve the initial altitude of the two contact points with respect to the ground. This basic expectation is confirmed by numerical simulations. For the purpose of its illustration, we consider a case where the bicycle simulation is started in a straight pitched configuration, such that $r_{1}(0)=0$ and ${ }^{0} s_{e}(t=0)=\left(-c \alpha_{0}, s \alpha_{0}, 0\right)^{T}$, with an initial pitch angle of $\pi-\alpha_{0}=-10^{\circ}$. This pitched bicycle is thrown with an initial forward velocity $V=\left(V_{1}^{2}+V_{2}^{2}\right)^{1 / 2}=4.6 \mathrm{~m} / \mathrm{s}$. The simulation is performed on a duration of $5 \mathrm{~s}$ with a step of $0.001 \mathrm{~s}$. As shown in figure 10, the two contact points do keep their altitude

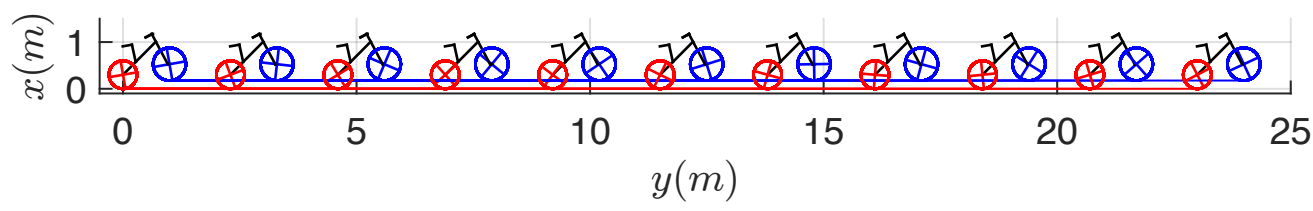

Figure 10: Snapshots in the $x-y$ plane, sampled along the path of a bicycle initialized with a non-zero pitch angle. The time interval between each snapshot is equal to $0.5 \mathrm{~s}$

constant along the motion (here in straight line). Going into details, the contact model (15) fed with the initial configuration $(g(0), r(0))$ calculates the two contact angles $\alpha_{0}$ and $\alpha_{1}$ which are used to calculate the space of admissible velocities $H$, these velocities ensuring each of the two wheels to roll on a parallel plane to the ground with a constant altitude. In the present case, because $\Phi_{p}(g(0), r(0)) \neq 0_{2}$, these planes do not coincide with the ground but have an altitude fixed by the initial conditions. Based on this insight, in all the following simulations, the reduced dynamics will be initialized with the statically balanced configuration pictured in figure 2, which trivially satisfies the two zero-altitude holonomic constraints (25). However, as this is mentioned by remark 5 , the numerical integration of the reconstruction equation introduces a slight drift of the constraints (25) which makes progressively the bicycle lift off the ground. Though being small, this drift can be removed by replacing the reconstruction equation of (66) by its corrected version:

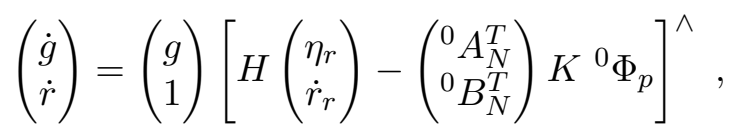

where $K$ is a $6 \times 6$ positive matrix of high gains, and ${ }^{0} \Phi_{p}$ and $\left({ }^{0} A_{N},{ }^{0} B_{N}\right)$ model the geometric and kinematic constraints imposed by the 2 non-penetration nor detachment conditions of the wheels along the normal to the ground, but expressed in the reference frame $\mathcal{F}_{0}$. To derive $\left({ }^{0} A_{N},{ }^{0} B_{N}\right)$ and ${ }^{0} \Phi_{p}$ we can refer to $(24)$ and (60), and write:

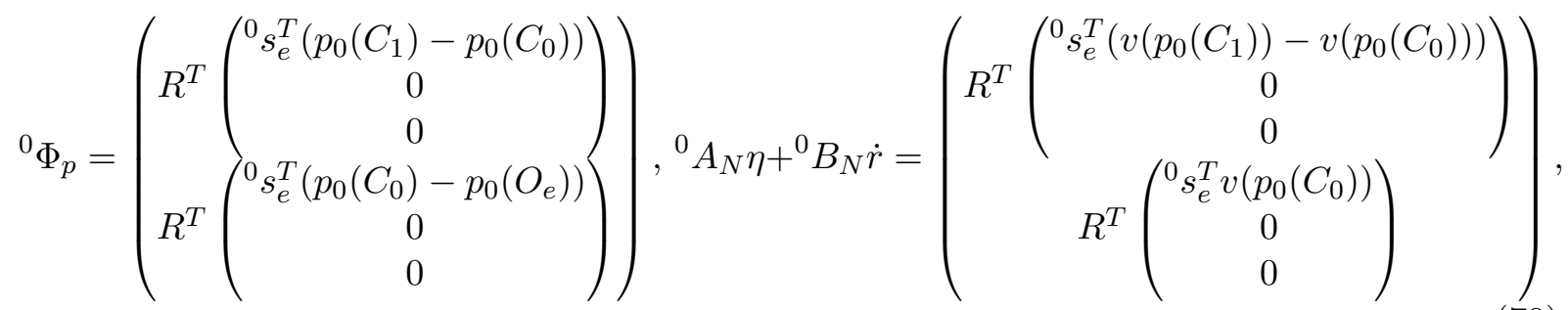

where the factors of ${ }^{0} s_{e}^{T}$ can be easily expressed in terms of $(g, r)$ and $(\eta, \dot{r})$ by using $(24)$ and (37). Now, if the components of $K$ are enough high, the corrected dynamics, i.e., the set of differential equations (66) with a dynamic stage unchanged and a kinematic stage replaced by the corrected reconstruction equation (69), can be interpreted as a two-time scales system with $(g, r)$ and $\left(\eta_{r}, \dot{r}_{r}\right)=\left(\Omega_{2}, \dot{r}_{1}, \dot{r}_{2}\right)$ the fast and slow components respectively [29]. Hence, the $(g, r)$-component is steered very quickly toward the slow manifold $\Phi_{p}=0_{2}$, while a slow 
motion takes place on the slow manifold according to the uncorrected dynamics (66). In short, the high-gain correction term in (69) ensures the numerical satisfaction of the two holonomic constraints without perturbing the bicycle dynamics. In practice we took for all the subsequent simulations:

$$
K=\frac{1}{\epsilon}\left(\begin{array}{cc}
10 \operatorname{Id}_{3 \times 3} & 0 \\
0 & \operatorname{Id}_{3 \times 3}
\end{array}\right),
$$

where $\epsilon$ is a small tuning parameter that we fixed to $\epsilon=0.01$.

\begin{tabular}{|l|r||l|r|}
\hline Parameter & Value & Parameter & Value \\
\hline$m$ & 6 & $n$ & 4 \\
$l_{1}$ & $0.986 \mathrm{~m}$ & $\gamma_{g}$ & $9.81 \mathrm{~m} \mathrm{~s}^{-2}$ \\
$l_{2}$ & $0.0337 \mathrm{~m}$ & $I_{2 x x}=I_{2 y y}$ & $0.141 \mathrm{~kg} \mathrm{~m}^{2}$ \\
$\mu$ & $\pi / 10 \mathrm{rad}$ & $I_{2 z z}$ & $0.28 \mathrm{~kg} \mathrm{~m}^{2}$ \\
$h_{2}$ & $0.35 \mathrm{~m}$ & $I_{3 x x}=I_{3 y y}$ & $0.0603 \mathrm{~kg} \mathrm{~m}^{2}$ \\
$h_{3}$ & $0.3 \mathrm{~m}$ & $I_{3 z z}$ & $0.12 \mathrm{~kg} \mathrm{~m}^{2}$ \\
$x_{0}$ & $0.6 \mathrm{~m}$ & $I_{0 x x}$ & $2.8 \mathrm{~kg} \mathrm{~m}^{2}$ \\
$y_{0}$ & $0.3 \mathrm{~m}$ & $I_{0 y y}$ & $9.2 \mathrm{~kg} \mathrm{~m}^{2}$ \\
$x_{1}$ & $0.0288 \mathrm{~m}$ & $I_{0 z z}$ & $11.0 \mathrm{~kg} \mathrm{~m}^{2}$ \\
$z_{1}$ & $0.368 \mathrm{~m}$ & $I_{0 x y}$ & $-2.4 \mathrm{~kg} \mathrm{~m}^{2}$ \\
$m_{0}$ & $85 \mathrm{~kg}$ & $I_{1 x x}$ & $0.0584 \mathrm{~kg} \mathrm{~m}^{2}$ \\
$m_{1}$ & $4 \mathrm{~kg}$ & $I_{1 y y}$ & $0.06 \mathrm{~kg} \mathrm{~m}^{2}$ \\
$m_{2}$ & $3 \mathrm{~kg}$ & $I_{1 z z}$ & $0.0076 \mathrm{~kg} \mathrm{~m}^{2}$ \\
$m_{3}$ & $2 \mathrm{~kg}$ & $I_{1 z x}$ & $-0.0091 \mathrm{~kg} \mathrm{~m}^{2}$ \\
\hline
\end{tabular}

Table 1: Simulation parameters.

\subsection{Passive motion on a tilted plane}

In this first dynamical example, the bicycle is passive (i.e., $\tau_{1}=\tau_{2}=\tau_{3}=0$ ) and thrown in its straight vertical configuration with an initial forward velocity of $V_{2}=4.6 \mathrm{~m} / \mathrm{s}$ along a planar ground tilted of $10^{\circ}$. The simulation is performed on a duration of $4 \mathrm{~s}$ whose the first $2.5 \mathrm{~s}$ are visualised in figure 11 with snapshots sampled every $0.5 \mathrm{~s}$. The bicycle first ascends the slope while decelerating. It stops around $t=3.40 \mathrm{~s}$, and then descends down the slope while accelerating. The ground slope is accounted by tilting the gravity field in the ground frame. The reconstruction equation being initialized in the straight vertical configuration with $\left(\alpha_{0}, \alpha_{1}\right)=(\pi, \mu+\pi)$, the steer and lean dynamics are structurally decoupled from the rolling ones and the excitation of the rolling dynamics does not affect the two others. Thus, the bicycle remains straight and vertical throughout the simulation.

\subsection{Passive asymptotic stabilisation of a bicycle}

For this second dynamic example, we have chosen to reproduce the simulation presented in [30]. It is related to the asymptotic stabilisation of a passive bicycle, i.e., as in the previous test $\tau_{1}=\tau_{2}=\tau_{3}=0$. To that end, the initial conditions are chosen as follows. The speed along $n_{0}$ (forward axis) is fixed to $V_{2}=4.6 \mathrm{~m} \mathrm{~s}^{-1}$ while the angular speed around $n_{0}$ (roll axis) is fixed to $\Omega_{2}=0.5 \mathrm{rads}^{-1}$. A top view of the bicycle motion is displayed in figure 12. Figure 13 presents the plots of the time evolution of the rolling velocity $\Omega_{2}$ and forward velocity $V_{2}$ of the frame, as well as the angular velocity of the handlebar $\dot{r}_{1}$ for the same test. Starting from their initial values, the plots of figure 13, which exactly fit with the numerical results provided in [30], clearly show that the bicycle self-stabilizes along time. Moreover, it can be 

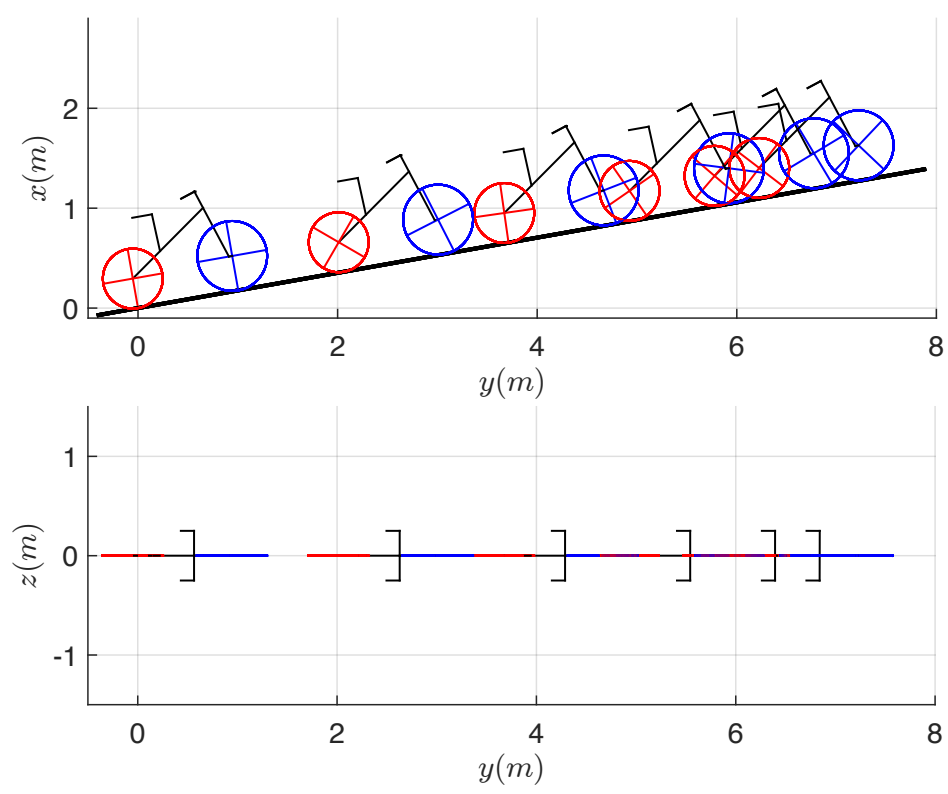

Figure 11: Snapshots along the ascending phase of a passive bicycle thrown on a tilted ground.

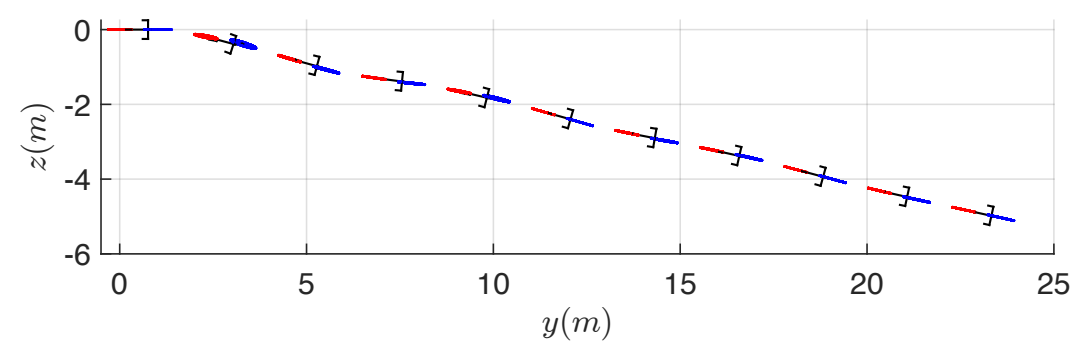

Figure 12: Trajectory of the bicycle in the $y$ - $z$ plane, in the context of the asymptotic stabilisation study case. The time interval between each snapshot is equal to $0.5 \mathrm{~s}$

noticed that, while the time runs, the values of $\Omega_{2}$ and $\dot{r_{1}}$ tend to zero while the forward speed of the frame increases up to reach a constant value closed to $V_{2}=4.622 \mathrm{~m} \mathrm{~s}^{-1}$ which is greater than the initial condition. As mentioned by [30], this is due to the conservation of the total energy of the system, a property satisfied by the simulation up to machine precision. Figure 15 and 16 show the angular positions of contacts $\alpha_{0}$ and $\alpha_{1}$ on the rear and front wheels respectively. Along simulation, due to the tilt of the bicycle frame, the two contact points first move along the wheels periphery before recovering their initial position (indicated by the red dotted line in the figures) when the bicycle converges toward its stable straight, vertical configuration. The simulator offers also the possibility to compute the Lagrange multipliers associated to the constraints. In our case, there are six multipliers ( 3 per wheel). For the purpose of illustration, figures 20 shows the time evolution of $\lambda_{4}$ which represents the force exerted along the axis $s_{0}$, onto the rear wheel at the contact point. This reaction force ensures the non-penetration of the rear wheel into the ground. Let us note that the multipliers are all expressed in the frame $\mathcal{F}_{0}$ (the bicycle frame) but can be easily expressed into the ground frame, for the purpose of ground-tires interactions study, for instance.

Finally, we close this example by a brief study of the drift and its correction. Figure 7.3 displays the time-evolution of the altitude of the two contact points with and without correction when $\epsilon=0.01$ and a time step of $0.001 \mathrm{~s}$. As expected, with no correction, the plots of figure 7.3 , which 


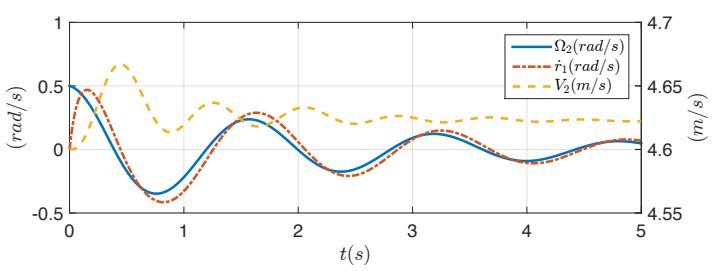

Figure 13: The rolling speed $\Omega_{2}$, the forward speed $V_{2}$ and the handlebar speed $\dot{r}_{1}$ versus time $t$.

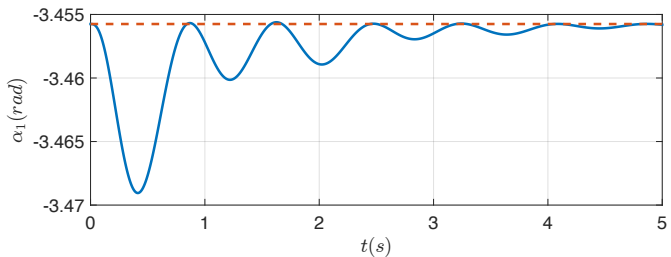

Figure 15: Time-evolution of the angular position of the contact point on the front wheel (i.e. $\alpha_{1}$ ) for the asymptotic stabilisation study case.

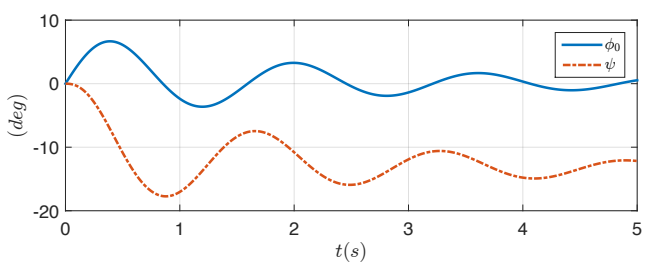

Figure 14: The roll angle $\phi_{0}$ and the yaw angle $\psi$ versus time $t$.

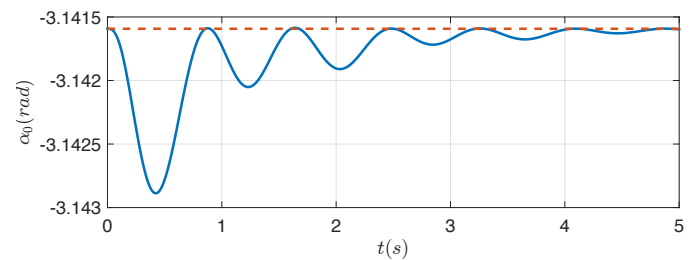

Figure 16: Time-evolution of the angular position of the contact point on the rear wheel (i.e. $\alpha_{0}$ ) for the asymptotic stabilisation study case.

are representative of all our simulations, show a slight drift of the contact points altitude which increases closely linearly with time. The second column of the table 7.3 gives the averaged vertical velocity $<{ }^{0} s_{e}^{T} v\left(p_{0}\left(C_{1}\right)\right)>$ of the front wheel contact point $C_{1}$ as a function of the integration time-step. As expected, the drift decreases with the time step and is very small for reasonably small time steps. Note also that with $\epsilon=0.01$, the drift of figure 7.3 remains is in the order of $10^{-12} \mathrm{~m}$ which for a time-step of $0.001 \mathrm{~s}$ falls into the precision range of our 4-order integrator. Finally, as expected, the drift correction does not affect the time evolution of the slow dynamics as this is illustrated by the plots of figures 7.3 and 19 which show that the corrected and uncorrected slow variables $\Omega_{2}$ and $\dot{r}_{1}$, fit up to 10 digits.

\subsection{Passive motion and falling on a tilted plane}

In this third dynamical example, the bicycle is thrown in the conditions of test 2 , except that $r_{1}$ is now initialized with a very small non-zero value $r_{1}=10^{-8} \mathrm{rad}$. This introduces a very slight asymmetry in the configuration that suffices to cause the falling of the bicycle. In fact, while the bicycle first ascends the slope as in the test 2, when stoping, instead of descending with

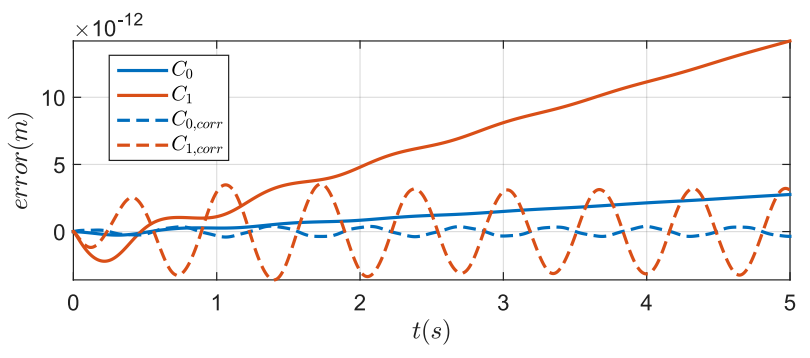

Figure 17: Time-evolution of the altitude of the contact points $C_{0}$ and $C_{1}$ in the example of the self-stabilization with (dashed line) and without (solid line) correction of the drift. Time-step of integration: $0.001 \mathrm{~s}, \epsilon=0.01$. 


\begin{tabular}{|c||c|}
\hline Time-step (s) & $<{ }^{0} s_{e}^{T} v\left(p_{0}\left(C_{1}\right)\right)>(\mu \mathrm{m} / \mathrm{s})$ \\
\hline 0,01 & $0.0655(1 \mathrm{~mm}$ for 4.24 hours $)$ \\
0,005 & $0.0030815(1 \mathrm{~mm}$ for 3.76 days $)$ \\
0,0025 & $0.00015257(1 \mathrm{~mm}$ for 2.59 months $)$ \\
0,001 & $0.00000321(1 \mathrm{~mm}$ for 9.87 years $)$ \\
0,0005 & $0.00000018434(1 \mathrm{~mm}$ for 171.9 years $)$ \\
\hline
\end{tabular}

Table 2: Drift velocity of the front wheel contact point $C_{1}$ as a function of the integration time-step for the passive asymptotic stabilisation example.

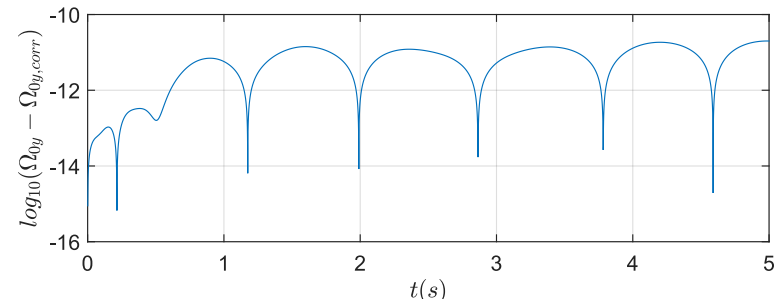

Figure 18: Decimal logarithm of the difference between $\Omega_{2}$ with, and without drift correction $(\epsilon=0.01)$.

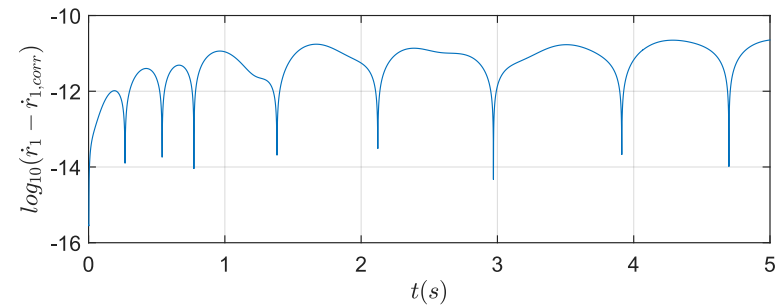

Figure 19: Decimal logarithm of the difference between $\dot{r}_{1}$ with, and without drift correction $(\epsilon=0.01)$.

its straight vertical configuration $\left(r_{1}=0\right)$, its configuration brutally tilts while its handlebar quickly turns, and the bicycle falls. Since the constraints are persistent (not intermittent), when falling, the two wheels cannot lift off the ground. As shown in figure 23, all along the simulation (including the falling phase), the total mechanical energy $E$ is conserved to preserve its initial value $E=T+U=1870$ Joules in our case. When falling, since the bicycle takes full threedimensional configurations, the two contact points quickly shift away from their initial positions (see the ending parts of the two plots of figures 21 and 22).

\subsection{Controlled turn manoeuver}

\begin{tabular}{|l|r||l|r|}
\hline Parameter & Value & Parameter & Value \\
\hline$K_{p s}$ & $10 \mathrm{Nm}$ & $K_{i s}$ & $35 \mathrm{Nm} . \mathrm{s}^{-1}$ \\
$K_{p d}$ & $20 \mathrm{Nms}$ & $K_{i d}$ & $100 \mathrm{Nm}$ \\
$r_{1 d}$ & $\pi / 36 \mathrm{rad}$ & $\dot{r}_{3 d}$ & $20{\mathrm{rad} . \mathrm{s}^{-1}}$ \\
$t_{1}$ & $0 \mathrm{~s}$ & $t_{2}$ & $5 \mathrm{~s}$ \\
\hline
\end{tabular}

Table 3: The simulation parameters of the controlled turn manoeuver.

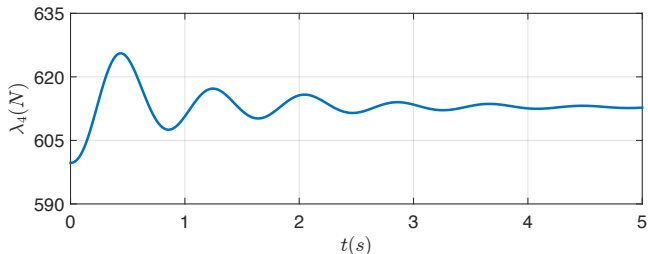

Figure 20: Time evolution of $\lambda_{4}$ the force applied on the rear wheel prohibiting the penetration and lift of the wheel in the ground.

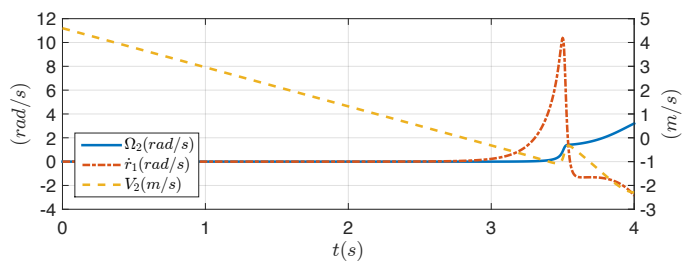

Figure 21: Time evolution of $V_{2}, \Omega_{2}$ and $\dot{r}_{1}$ of a bicycle rolling on a tilted ground. 


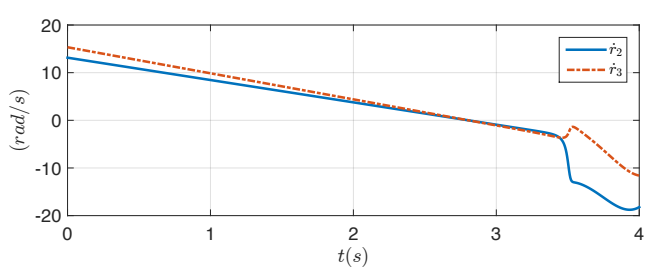

Figure 22: Time evolution of $\dot{r}_{2}$ and $\dot{r}_{3}$ of a bicycle rolling on a tilted ground.

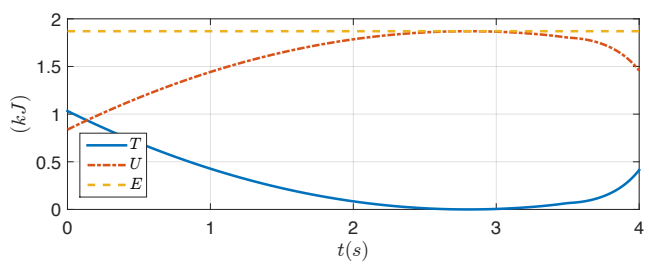

Figure 23: Time evolution of the kinetic, potential and total energy of a bicycle rolling on a tilted ground.

In this last numerical example, the handlebar and the rear wheel are actuated to ensure the bicycle to perform a turn at a controlled forward speed. To do that, we use the simple proportionalintegral torque control law:

$$
e_{1}(t)=f_{s}\left(t, t_{1}, t_{2}\right) r_{1 d}-r_{1}, \text { and } \tau_{1}(t)=-K_{p s} e_{1}(t)-K_{i s} \int_{0}^{t} e_{1}(\xi) d \xi,
$$

where, $K_{p s}$ is a steering proportional gain, $K_{i s}$ is a steering integral gain, $r_{1 d}$ is the desired steering angle, $t_{1} \leq t_{2}$ are switching times used to perform a curved path, and $f_{s}\left(t, t_{i}, t_{f}\right)$ is a slope function defined as follows:

If $t \leq t_{i}$ :

$$
f_{s}=0
$$

Else if $t_{i}<t \leq t_{f}$ :

$$
f_{s}=\frac{t-t_{i}}{t_{f}-t_{i}}-\frac{1}{2 \pi} \sin \left(2 \pi \frac{t-t_{i}}{t_{f}-t_{i}}\right)
$$

Else:

$$
f_{s}=1
$$

End.

Where $t_{i}$ and $t_{f}$ are the starting and ending times of the slope respectively.

Similarly, the torque applied on the hub of the rear wheel is defined as:

$$
e_{3}=\dot{r}_{3 d}-\dot{r}_{3}, \text { and } \tau_{3}=K_{p d} e_{3}+K_{i d} \int_{0}^{t} e_{3}(\xi) d \xi,
$$

where $K_{p d}, K_{i d}$ stand for a proportional and an integral gain, while $\dot{r}_{3 d}$ is the desired rear wheel speed. The values of the controller parameters are indicated in table 3.

As shown in figure 24, the bicycle starts from a straight vertical configuration with an initial forward velocity $V_{2}=\dot{r}_{3 d} h_{3}=6 \mathrm{~m} . \mathrm{s}^{-1}$, and performs a turn with a prescribed radius of $11.6 \mathrm{~m}$ and a prescribed constant forward speed (see figure 27). When the handlebar starts to increase following the slop from $r_{1}=0$ to $r_{1} \cong r_{1 d}$ (see figure 26), the bicycle naturally (passively) tilts toward the center of the curve while turning. To illustrate this, we plotted in figure 28 the time evolution of the rolling, steering, and forward velocities which stabilize with time. As far as the actuation torques are concerned, figure 29 and figure 30 show the time evolution of the torques applied to the handlebar $\left(\tau_{1}\right)$, and to the rear hub $\left(\tau_{3}\right)$. Note that to initiate the turn, the biker needs to apply a torque of opposite sign with respect to that of the steering angle. On the other hand, once the desired steering angle is reached, to maintain its turning the torque and the steering angle have the same sign. The time-evolution of the angular position of the contact point between the front and rear wheels and the ground, are plotted in figure 31 and 32 respectively. Let us note that during the maneuver, the contact point migrates forward along the front wheel to reach $0.04 \mathrm{rad} \cong 2.3^{\circ}$. 


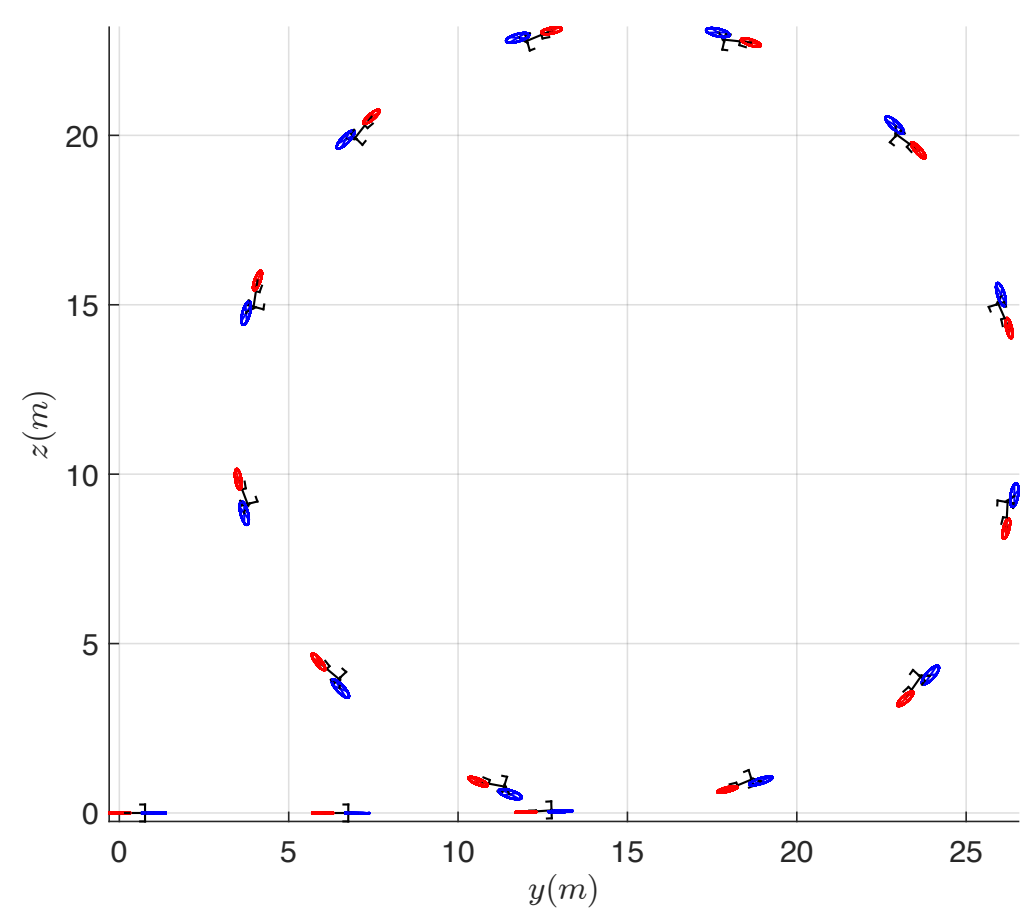

Figure 24: Trajectory of the bicycle in the $y-z$ plane when performing a controlled turn. The time interval between each snapshot is equal to $1 \mathrm{~s}$.

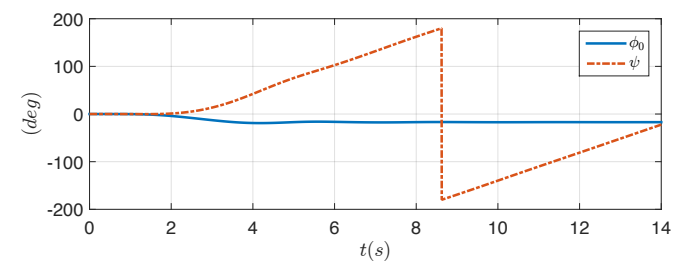

Figure 25: The roll angle $\phi_{0}$ and the yaw angle $\psi$ versus time $t$.

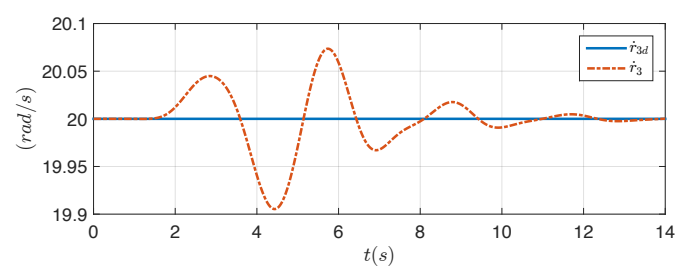

Figure 27: Time-evolution of the rear wheel speed $\dot{r}_{3}$ versus the setpoint $\dot{r}_{3 d}$.

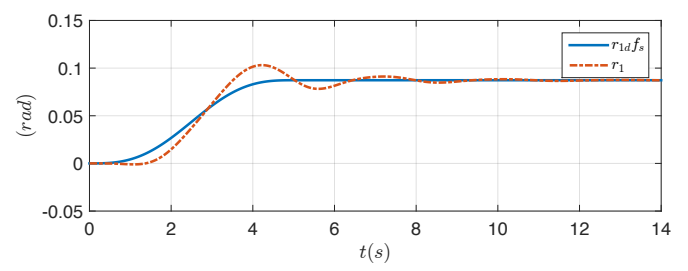

Figure 26: Time-evolution of the steering angle $r_{1}$ versus the desired value $f_{s} r_{1 d}$.

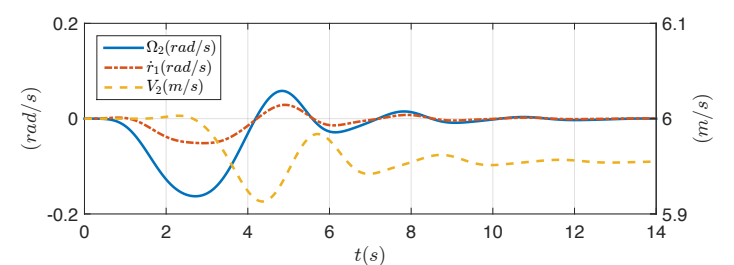

Figure 28: Time-evolution of the rolling (in blue), steering (in red), and forward (in yellow) velocities during the turn manoeuver.

\section{Conclusion}

In this article, we proposed a new formulation of the dynamics of the Whipple bicycle. The approach is based on the geometric mechanics of locomotion multibody systems in their principal fiber bundle of configuration $G \times S$. Exploiting the intrinsic character of geometric mechanics, most of the geometric nonlinearities met in the usual model of the same system, but expressed in coordinates, are here shifted to the numerical integration of a set of reconstruction equations from 


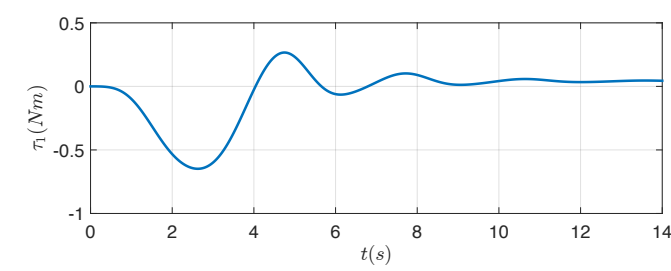

Figure 29: Time-evolution of the steering torque $\tau_{1}$.

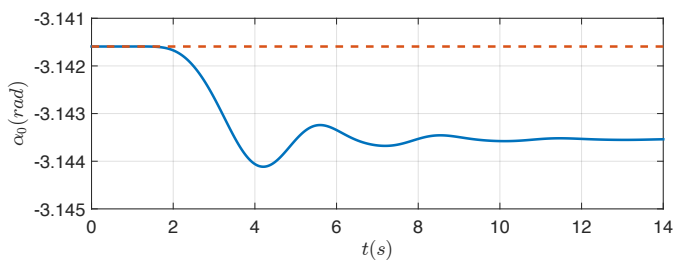

Figure 31: Time-evolution of the angular position of the contact point on the rear wheel (i.e. $\alpha_{0}$ ) during the turn manoeuver.

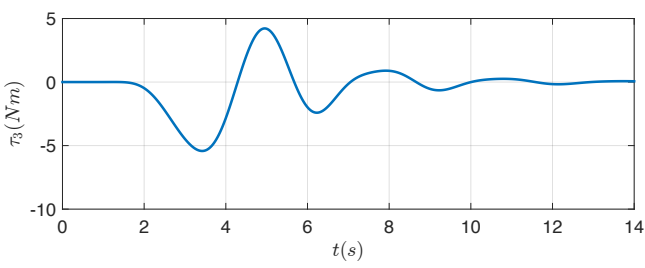

Figure 30: Time-evolution of the driving torque $\tau_{3}$.

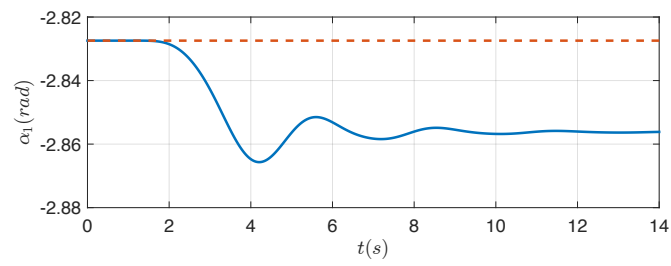

Figure 32: Time-evolution of the angular position of the contact point on the front wheel (i.e. $\alpha_{1}$ ) during the turn manoeuver.

the space of admissible velocities to $G \times S$. Based on this idea, we used $G=S E(3)$ to remove the three-dimensional geometric nonlinearities of the bicycle and replaced its two holonomic constraints of non lifting nor penetration, by their kinematic form in terms of non-integrable velocities. This allowed us to derive the close form of the full nonlinear dynamics of the bicycle in the form of a set of reduced dynamics equations in the kernel of the constraints imposed by the contacts between the wheels and the ground. These equations are exact for any initial configuration of the bicycle satisfying the two holonomic constraints. The derivation of these equations is performed by following a multistage general modelling algorithm which starts with the geometric model of contacts, and continues with the implicit kinematic model of constraints, the explicit kinematic model or reduced kinematics, and finally the dynamic model of the system free of constraints in $S E(3) \times S$ and its projection in the kernel of constraints. As a result, the article contributes to illustrate this general framework on a non-trivial concrete system while giving an alternative formulation of the Whipple bicycle dynamics. From that perspective, we attempted to privilege symbolic derivations over numerical solutions. In the end, the reduced dynamics have been obtained in a closed symbolic form and numerically integrated on several tests representative of the bicycle dynamics including the well known test of self-stabilization. These further numerical simulations show that the approach does preserve the constraints and the energy as this is expected for this system.

\section{Appendix 1: Calculation of the kernel of constraints}

In this appendix, we calculate $H=\operatorname{ker}(A, B)$ by inverting symbolically the system that we rewrite in the form $D=(A, B)\left(\eta^{T}, \dot{r}^{T}\right)^{T}=0_{6}$ where $D$ is the $6 \times 1$ vector whose components $D_{i}, i=1,2 \ldots 6$, form the left hand side of (38-43). Firstly, $V_{1}, V_{2}$ and $V_{3}$ can be extracted from $D_{4}, D_{5}$ and $D_{6}$ respectively, as follows:

$$
\begin{aligned}
& V_{1}=F_{16} \Omega_{3}+F_{19} \dot{r_{3}}, \\
& V_{2}=F_{26} \Omega_{3}+F_{29} \dot{r_{3}}, \\
& V_{3}=F_{34} \Omega_{1}+F_{35} \Omega_{2},
\end{aligned}
$$


where we introduced the notations:

$$
\begin{aligned}
& F_{16}=-A_{46}, F_{19}=-B_{43}, \\
& F_{26}=-A_{56}, F_{29}=-B_{53}, \\
& F_{34}=-A_{64}, F_{35}=-A_{65} .
\end{aligned}
$$

After inserting (79) in $D_{3}$, one can express $\Omega_{1}$ as:

$$
\Omega_{1}=F_{45} \Omega_{2}+F_{47} \dot{r_{1}}+F_{48} \dot{r_{2}},
$$

with:

$$
F_{45}=-\frac{A_{35}+F_{35}}{F_{34}+A_{34}}, F_{47}=-\frac{B_{31}}{F_{34}+A_{34}}, F_{48}=-\frac{B_{32}}{F_{34}+A_{34}} .
$$

In a similar way, inserting $(77)$ in $D_{1}$, gives:

$$
\dot{r_{2}}=F_{85} \Omega_{2}+F_{86} \Omega_{3}+F_{87} \dot{r_{1}}+F_{89} \dot{r_{3}},
$$

with:

$$
F_{85}=-\frac{A_{15}}{B_{12}}, F_{86}=-\frac{A_{16}+F_{16}}{B_{12}}, F_{87}=-\frac{B_{11}}{B_{12}}, F_{89}=-\frac{F_{19}}{B_{12}} .
$$

Inserting (85), (77) and (83) in $D_{2}$, allows rewriting $\Omega_{3}$ as:

$$
\Omega_{3}=H_{65} \Omega_{2}+H_{67} \dot{r_{1}}+H_{69} \dot{r_{3}},
$$

with:

$$
\begin{gathered}
H_{65}=-\frac{A_{24} F_{45}+\left(A_{24} F_{48}+B_{22}\right) F_{85}}{\left(B_{22}+A_{24} F_{48}\right) F_{86}+F_{26}+A_{26}}, H_{67}=-\frac{B_{21}+A_{24} F_{47}+\left(A_{24} F_{48}+B_{22}\right) F_{87}}{\left(B_{22}+A_{24} F_{48}\right) F_{86}+F_{26}+A_{26}}, \\
H_{69}=-\frac{F_{29}+\left(A_{24} F_{48}+B_{22}\right) F_{89}}{\left(B_{22}+A_{24} F_{48}\right) F_{86}+F_{26}+A_{26}} .
\end{gathered}
$$

Now, inserting (87) in (77), (78) and (85) allows us to rewrite them in the following form:

$$
\begin{aligned}
& V_{1}=H_{15} \Omega_{2}+H_{17} \dot{r_{1}}+H_{19} \dot{r_{3}}, \\
& V_{2}=H_{25} \Omega_{2}+H_{27} \dot{r_{1}}+H_{29} \dot{r_{3}}, \\
& \dot{r_{2}}=H_{85} \Omega_{2}+H_{87} \dot{r_{1}}+H_{89} \dot{r_{3}},
\end{aligned}
$$

with:

$$
\begin{gathered}
H_{15}=F_{16} H_{65}, H_{15}=F_{16} H_{65}, H_{19}=F_{16} H_{69}+F_{19}, \\
H_{25}=F_{26} H_{65}, H_{27}=F_{26} H_{67}, H_{29}=F_{26} H_{69}+F_{29}, \\
H_{85}=F_{86} H_{65}+F_{85}, H_{87}=F_{86} H_{67}+F_{87}, H_{89}=F_{86} H_{69}+F_{89} .
\end{gathered}
$$

Then, using (92) in (83) gives:

$$
\Omega_{1}=H_{45} \Omega_{2}+H_{47} \dot{r_{1}}+H_{49} \dot{r_{3}},
$$

with:

$$
H_{45}=F_{48} H_{85}+F_{45}, H_{47}=F_{48} H_{87}+F_{47}, H_{49}=F_{48} H_{89} .
$$

Finally, inserting (96) into (79), we find:

$$
V_{3}=H_{35} \Omega_{2}+H_{37} \dot{r_{1}}+H_{39} \dot{r_{3}},
$$

with:

$$
H_{35}=F_{34} H_{45}+F_{35}, H_{37}=F_{34} H_{47}, H_{39}=F_{34} H_{49} .
$$

Finally, any vector in the kernel of the constraints can be written in the form (62) which require the ordered calculations of (80), (81), (82), (84), (86), (88), (89), (93), (94), (95), (97), (99). 


\section{Appendix 2: Free bicycle dynamics}

We first start by calculating the acceleration of the four bodies that compose the bicycle in the configuration space $S E(3) \times S$. This is done by removing all the accelerations $\dot{\eta}_{j}$, for $j \neq 0$ in the recursion on accelerations (64). After straightforward algebra we find:

$$
\begin{aligned}
& \dot{\eta_{0}}=\dot{\eta}_{0}, \\
& \dot{\eta_{1}}=\operatorname{Ad}_{g_{0}} \dot{\eta}_{0}+\ddot{r}_{1} \mathrm{~A}_{1}+\zeta_{1}, \\
& \dot{\eta_{2}}=\operatorname{Ad}_{2} \dot{g}_{0} \dot{\eta}_{0}+\operatorname{Ad}_{2}{ }_{g_{1}} \mathrm{~A}_{1} \ddot{r_{1}}+\mathrm{A}_{2} \ddot{r_{2}}+\operatorname{Ad}_{2}{ }_{g_{1}} \zeta_{1}+\zeta_{2}, \\
& \dot{\eta_{3}}=\operatorname{Ad}_{g_{0}} \dot{\eta_{0}}+\mathrm{A}_{3} \ddot{r_{3}}+\zeta_{3},
\end{aligned}
$$

which need to use the detail expressions of the adjoint map and its time-derivative:

$$
\operatorname{Ad}_{j g_{i}}=\left(\begin{array}{cc}
{ }^{j} R_{i} & { }^{j} R_{i} \hat{p}_{i}\left(O_{j}\right)^{T} \\
0 & { }^{j} R_{i}
\end{array}\right), \zeta_{j}=\left(\begin{array}{c}
\left({ }^{j} V_{i}+p_{j}\left(O_{i}\right) \times{ }^{j} \Omega_{i}\right) \times \dot{r}_{j} a_{j} \\
\Omega_{i} \times \dot{r}_{j} a_{j}
\end{array}\right),
$$

and where $\mathrm{A}_{j}=\left(0_{3}^{T}, a_{j}^{T}\right)^{T}$ is the $(6 \times 1)$ unit vector supporting the joint axis $j$. Now writing the top row of (63) with $k$ the indexes of all the bodies just after $\mathcal{B}_{j}$ when descending the structure from $\mathcal{B}_{0}$ to its tips, we have for each of the four bodies of the bicycle:

$$
\begin{aligned}
& f_{3}=\mathcal{M}_{3} \dot{\eta}_{3}+f_{i n, 3}+f_{\text {ext }, 3}, \\
& f_{2}=\mathcal{M}_{2} \dot{\eta}_{2}+f_{\text {in, } 2}+f_{\text {ext }, 2}, \\
& f_{1}=\mathcal{M}_{1} \dot{\eta}_{1}+f_{\text {in, } 1}+f_{\text {ext }, 1}+\operatorname{Ad}_{2}^{T} f_{1}, \\
& f_{0}=\mathcal{M}_{0} \dot{\eta}_{0}+f_{\text {in }, 0}+f_{\text {ext }, 0}+\operatorname{Ad}_{3}^{T} f_{0} f_{3}+\operatorname{Ad}_{1}^{T} f_{0} f_{1},
\end{aligned}
$$

which need the detailed expressions:

$$
\mathcal{M}_{j}=\left(\begin{array}{cc}
m_{j} 1_{3 \times 3} & \hat{m} s_{j}^{T} \\
\hat{m} s_{j} & I_{j}
\end{array}\right), f_{i n, j}=\left(\begin{array}{c}
\left(m s_{j} \times \Omega_{j}\right) \times \Omega_{j}+\Omega_{j} \times\left(m_{j} V_{j}\right) \\
\Omega_{j} \times\left(I_{j} \Omega_{j}\right)+m s_{j} \times\left(\Omega_{j} \times V_{j}\right)
\end{array}\right),
$$

where $m_{j} 1_{3 \times 3}$ and $I_{j}$ are the matrices of linear and angular inertia while $m s_{j}=m_{j} p_{j}\left(G_{j}\right)$ is the vector of first inertia moments that couple linear and angular accelerations, all being related to $\mathcal{B}_{j}$. Then, inserting (107), (106), (105), (101), (102) and (103) into (108) that we identify with the first row of $(65)$, we obtain:

$$
\begin{aligned}
\mathcal{M} & =\sum_{i=0}^{3} \operatorname{Ad}_{i_{g_{0}}}^{T} \mathcal{M}_{i} \operatorname{Ad}_{i_{g_{0}}}, \\
M^{T} & =\left(\sum_{i=1}^{2} \operatorname{Ad}_{i_{g_{0}}}^{T} \mathcal{M}_{i} \operatorname{Ad}_{i_{g_{1}}} \mathrm{~A}_{1}, \operatorname{Ad}_{2}^{T} g_{g_{0}} \mathcal{M}_{2} \mathrm{~A}_{2}, \operatorname{Ad}_{3}^{T}{ }_{g_{0}} \mathcal{M}_{3} \mathrm{~A}_{3}\right), \\
f_{\text {in }} & =\sum_{i=0}^{3} \operatorname{Ad}_{i g_{0}}^{T} f_{i n, i}+\sum_{j=1}^{2}\left(\sum_{i=j}^{2} \operatorname{Ad}_{i g_{0}}^{T} \mathcal{M}_{i} \operatorname{Ad}_{i_{j}}\right) \zeta_{j}+\operatorname{Ad}_{3}^{T}{ }_{g_{0}} \mathcal{M}_{3} \zeta_{3}, \\
f_{\text {ext }} & =\sum_{i=0}^{3} \operatorname{Ad}_{i_{g_{0}}}^{T} f_{\text {ext }, i} .
\end{aligned}
$$

Then introducing (105)-(108) into $\tau_{j}=\mathrm{A}_{j}^{T} f_{j}$, gives :

$$
\begin{aligned}
\tau_{1} & =\mathrm{A}_{1}^{T}\left(\mathcal{M}_{1} \dot{\eta_{1}}+f_{i n, 1}+f_{\text {ext }, 1}+\operatorname{Ad}_{2}^{T} g_{1}\left(\mathcal{M}_{2} \dot{\eta_{2}}+f_{i n, 2}+f_{\text {ext }, 2}\right)\right) \\
\tau_{2} & =\mathrm{A}_{2}^{T}\left(\mathcal{M}_{2} \dot{\eta}_{2}+f_{i n, 2}+f_{\text {ext }, 2}\right) \\
\tau_{3} & =\mathrm{A}_{3}^{T}\left(\mathcal{M}_{3} \dot{\eta_{3}}+f_{i n, 3}+f_{\text {ext }, 3}\right)
\end{aligned}
$$


that once identified with the second row of (65), gives the expressions below:

$$
\begin{aligned}
& m=\left(\begin{array}{ccc}
\sum_{i=1}^{2} \mathrm{~A}_{1}^{T} \operatorname{Ad}_{i_{g_{1}}}^{T} \mathcal{M}_{i} \operatorname{Ad}_{{ }_{g_{1}}} \mathrm{~A}_{1} & \mathrm{~A}_{1}^{T} \operatorname{Ad}_{2}^{T} \mathcal{M}_{2} \mathrm{~A}_{2} & 0 \\
\mathrm{~A}_{2}^{T} \mathcal{M}_{2} \mathrm{Ad}_{2} \mathrm{~g}_{1} \mathrm{~A}_{1} & \mathrm{~A}_{2}^{T} \mathcal{M}_{2} \mathrm{~A}_{2} & 0 \\
0 & 0 & \mathrm{~A}_{3}^{T} \mathcal{M}_{3} \mathrm{~A}_{3}
\end{array}\right), \\
& Q_{i n}=\left(\begin{array}{c}
\sum_{i=1}^{2} \mathrm{~A}_{1}^{T} \operatorname{Ad}_{i g_{1}}^{T} f_{i n, i}+\sum_{j=1}^{2}\left(\sum_{i=j}^{2} \mathrm{~A}_{1}^{T} \operatorname{Ad}_{i g_{1}}^{T} \mathcal{M}_{i} \operatorname{Ad}_{i_{j}}\right) \zeta_{j} \\
\mathrm{~A}_{2}^{T} f_{i n, 2}+\sum_{i=1}^{2} \mathrm{~A}_{2}^{T} \mathcal{M}_{2} \operatorname{Ad}_{2} \zeta_{i} \zeta_{i} \\
\mathrm{~A}_{3}^{T} f_{i n, 3}+\mathrm{A}_{3}^{T} \mathcal{M}_{3} \zeta_{3}
\end{array}\right), Q_{\text {ext }}=\left(\begin{array}{c}
\sum_{i=1}^{2} \mathrm{~A}_{1}^{T} \operatorname{Ad}_{i g_{1}}^{T} f_{\text {ext }, i} \\
\mathrm{~A}_{2}^{T} f_{\text {ext }, 2} \\
\mathrm{~A}_{3}^{T} f_{\text {ext }, 3}
\end{array}\right) .
\end{aligned}
$$

Finally, once supplemented with the recursive geometric model ${ }^{e} g_{j}={ }^{e} g_{i}{ }^{i} g_{j}\left(r_{j}\right)$ and the kinematic one (63-bottom), the above expressions along with (110-113) define all the matrices of the free dynamics, or equivalently, of the left-hand-side of (3) and (4).

\section{References}

[1] Appell, P.: Traité de mécanique rationnelle. Gauthier-Villars et Cie., Paris (1931)

[2] Astrom, K.J., Klein, R.E., Lennartsson, A.: Bicycle dynamics and control: adapted bicycles for education and research. IEEE Control Systems 25(4), 26-47 (2005)

[3] Aström, K.J., Murray, R.M.: Feedback systems: an introduction for scientists and engineers. Princeton university press (2010)

[4] Basu-Mandal, P., Chatterjee, A., Papadopoulos, J.: Hands-free circular motions of a benchmark bicycle. Proceedings of the Royal Society of London A: Mathematical, Physical and Engineering Sciences 463(2084), 1983-2003 (2007)

[5] Bloch, A.M.: Nonholonomic Mechanics and Control, Interdisciplinary Applied Mathematics, vol. 24. Springer-Verlag New York (2015)

[6] Bloch, A.M., Krishnaprasad, P.S., Marsden, J.E., Murray, R.M.: Nonholonomic mechanical systems with symmetry. Archive for Rational Mechanics and Analysis 136(1), 21-99 (1996)

[7] Bourlet, C.: Étude théorique sur la bicyclette. Bulletin de la Société Mathématique de France 27, 76-96 (1899)

[8] Boussinesq, J.: Aperçu sur la théorie de la bicyclette. Journal de Mathématiques Pures et Appliquées 5, 117-136 (1899)

[9] Boyer, F., Belkhiri, A.: Reduced locomotion dynamics with passive internal dofs: Application to nonholonomic and soft robotics. IEEE Transactions on Robotics 30(3), 578-592 (2014)

[10] Boyer, F., Belkhiri, A.: Erratum to "reduced locomotion dynamics with passive internal dofs: Application to nonholonomic and soft robotics" [jun 14 578-592]. IEEE Transactions on Robotics 31(3), 805-805 (2015)

[11] Boyer, F., Porez, M.: Multibody system dynamics for bio-inspired locomotion: from geometric structures to computational aspects. Bioinspiration \& Biomimetics 10(2), 1-21 (2015) 
[12] Boyer, F., Primault, D.: The Poincaré-Chetayev equations and flexible multibody systems. Journal of Applied Mathematics and Mechanics, http://hal.archives-ouvertes.fr/hal00672477 69(6), 925-942 (2005)

[13] Campion, G., Bastin, G., D'Andréa-Novel, B.: Structural properties and classification of kinematic and dynamic models of wheeled mobile robots. IEEE Transactions on Robotics and Automation 12(1), 47-62 (1996)

[14] Carvallo, E.: Théorie du movement du monocycle part 2: Théorie de la bicyclette. Journal de l'École Polytechnique 6, 1-118 (1901)

[15] Cendra, H., Marsden, J.E., Ratiu, T.S.: Mathematics Unlimited - 2001 and Beyond, chap. Geometric mechanics, Lagrangian reduction, and nonholonomic systems, pp. 221273. Springer-Verlag (2001)

[16] Chaplygin, S.: On the theory of motion of nonholonomic systems. the reducing-multiplier theorem. Regular and Chaotic Dynamics 13(4), 369-376 (2008)

[17] Chitta, S., Cheng, P., Frazzoli, E., Kumar, V.: Robotrikke: A novel undulatory locomotion system. In: Proceedings of the 2005 IEEE International Conference on Robotics and Automation (ICRA), pp. 1597-1602 (2005)

[18] Consolini, L., Maggiore, M.: Control of a bicycle using virtual holonomic constraints. Automatica 49(9), 2831-2839 (2013)

[19] Featherstone, R.: Rigid Body Dynamics Algorithms. Springer (2008)

[20] Franke, G., Suhr, W., Riess, F.: An advanced model of bicycle dynamics. European Journal of Physics 11(2), 116-121 (1990)

[21] Getz, N.H., Marsden, J.E.: Control for an autonomous bicycle. In: Proceedings of 1995 IEEE International Conference on Robotics and Automation, vol. 2, pp. 1397-1402 vol.2 (1995)

[22] Hertz, H.: Die Prinzipen der Mechanik in neuem Zusammenhange dargestellt. Gesamelte Werke, Band III. Leipzig (1894)

[23] Jones, A.T.: Physics and bicycles. American Journal of Physics 10(6), 332-333 (1942)

[24] Kelly, S.D., Murray, R.M.: Geometric phases and robotic locomotion. Journal of Robotic Systems 12(6), 417-431 (1995)

[25] Klein, F., Sommerfeld, A.: Über die theorie des kreisels. Über die Theorie des Kreisels, by Klein, Felix; Sommerfeld, Arnold. New York: Johnson Reprint Corp., 1965. Bibliotheca mathematica Teubneriana; Bd. 14 (1965)

[26] Kooijman, J.D.G., Meijaard, J.P., Papadopoulos, J.M., Ruina, A., Schwab, A.L.: A bicycle can be self-stable without gyroscopic or caster effects. Science 332(6027), 339-342 (2011)

[27] Le Hénaff, Y.: Dynamical stability of the bicycle. European Journal of Physics 8(3), 207$210(1987)$

[28] Letov, A.: Stability of an automatically controlled bicycle moving on a horizontal plane. Journal of Applied Mathematics and Mechanics 23(4), 934-942 (1959) 
[29] Lobry, C., Sari, T.: Singular perturbation methods in control theory. In: Contrôle Non Linéaire et Applications, Cours du CIMPA, Collection Travaux en Cours, pp. 155-182. Hermann, Paris (2005)

[30] Meijaard, J., Papadopoulos, J.M., Ruina, A., Schwab, A.: Linearized dynamics equations for the balance and steer of a bicycle: a benchmark and review. Proceedings of the Royal Society of London A: Mathematical, Physical and Engineering Sciences 463(2084), 1955$1982(2007)$

[31] Morin, P., Samson, C.: Control of nonholonomic mobile robots based on the transverse function approach. IEEE Transactions on Robotics 25(5), 1058-1073 (2009)

[32] Ostrowski, J., Burdick, J.: The geometric mechanics of undulatory robotic locomotion. The International Journal of Robotics Research (IJRR) 17(7), 683-701 (1998)

[33] Ostrowski, J., Burdick, J., Lewis, A.D., Murray, R.M.: The mechanics of undulatory locomotion: the mixed kinematic and dynamic case. In: Proceedings of 1995 IEEE International Conference on Robotics and Automation (ICRA), vol. 2, pp. 1945-1951 vol.2 (1995)

[34] Ostrowski, J., Lewis, A., Murray, R., Burdick, J.: Nonholonomic mechanics and locomotion: the snakeboard example. In: Proceedings of the 1994 IEEE International Conference on Robotics and Automation (ICRA), pp. 2391-2397 vol.3 (1994)

[35] Ostrowski, J.P.: Computing reduced equations for robotic systems with constraints and symmetries. IEEE Transactions on Robotics and Automation 15(1), 111-123 (1999)

[36] Psiaki, M.: Bicycle stability: A mathematical and numerical analysis. undergradute thesis. Physics Dept., Princeton University, NJ (1979)

[37] Rankine, W.J.M.: On the dynamical principles of the motion of velocipedes. The Engineer 28(79), 129 (1869)

[38] Timoshenko, S.P., Young, D.H.: Advanced dynamics. McGraw-Hill (1948)

[39] Whipple, F.J.: The stability of the motion of a bicycle. Quarterly Journal of Pure and Applied Mathematics 30(120), 312-348 (1899) 